Review

\title{
Synthetic Aspects and Electro-Optical Properties of Fluorinated Arylenevinylenes for Luminescence and Photovoltaics
}

\author{
Carmela Martinelli ${ }^{1}$, Gianluca M. Farinola ${ }^{2}$, Vita Pinto ${ }^{2}$ and Antonio Cardone ${ }^{1, *}$ \\ 1 Istituto di Chimica dei Composti OrganoMetallici CNR-ICCOM di Bari, Via Orabona 4, \\ Bari I-70125, Italy; E-Mail: martinelli.carmela@chimica.uniba.it \\ 2 Deparment of Chemistry, Università degli Studi di Bari —Ado Moro", Via Orabona 4, \\ Bari I-70125, Italy; E-Mails: gianlucamaria.farinola@uniba.it (G.M.F.); vita.pinto@uniba.it (V.P.) \\ * Author to whom correspondence should be addressed; E-Mail: cardone@ba.iccom.cnr.it;
} Tel./Fax: +39-080-5443-598.

Received: 14 February 2013; in revised form: 12 March 2013 / Accepted: 18 March 2013 / Published: 25 March 2013

\begin{abstract}
In this review, the main synthetic aspects and properties of fluorinated arylenevinylene compounds, both oligomers and polymers, are summarized and analyzed. Starting from vinyl organotin derivatives and aryl halides, the Stille cross-coupling reaction has been successfully applied as a versatile synthetic protocol to prepare a wide series of $\pi$-conjugated compounds, selectively fluorinated on the aromatic and/or vinylene units. The impact of fluoro-functionalization on properties, the solid state organization and intermolecular interactions of the synthesized compounds are discussed, also in comparison with the non-fluorinated counterparts. Luminescent and photovoltaic applications are also discussed, highlighting the role of fluorine on the performance of devices.
\end{abstract}

Keywords: arylenevinylenes; fluorinated materials; Stille cross-coupling; organic semiconductors; conjugated materials

\section{Introduction}

Current interest in the emerging fields of plastic electronics and photonics is based on the peculiar optical and semiconducting properties of organic $\pi$-conjugated materials, which can be designed and developed for applications in many opto-electronic devices [1-3], such as organic light-emitting diodes (OLEDs) [4-7] organic thin film transistors (OTFTs) [8,9], sensors [10] and photovoltaics [11-13]. 
The key advantages of organic semiconductors with respect to their inorganic counterparts reside in the possibility to fine-tailor properties on the molecular scale (e.g., band gap, HOMO and LUMO energy levels, charge mobility, thermal and chemical stability, processability) by proper design of the conjugated skeleton and functionalization, thus enabling the use of low-cost technologies in the fabrication of organic-based devices.

From a synthetic point of view, the functionalization of organic compounds with appropriate substituents plays a key role in the control and tuning of structural and chemico-physical properties. As a functional group, fluorine possesses a unique and extraordinary capability to modify the properties of the materials [14-16] through various effects. Deep investigations on these features often reveal even more interesting and unexpected results. The main effects of fluorine are related to the special characteristics of this small atom, such as the highest electronegativity (in the Pauling scale, EN = 4) and the high $\mathrm{C}-\mathrm{F}$ bond energy (about $480 \mathrm{KJ} / \mathrm{mol}$ ) in the periodic table. The strength of the $\mathrm{C}-\mathrm{F}$ bond improves the thermal and chemical stability of fluorinated organic compounds, which can result in an enhancement of the devices life-time. The electron-withdrawing effect of the fluorine atoms causes a lowering of the HOMO and LUMO energy levels of organic semiconductors, facilitating electron injection from metal electrodes and improving negative charge transport capability. As a consequence, fluorinated compounds behave as ambipolar or n-type semiconductors and can find useful application as active materials in bipolar or n-type OTFT [17-22]. Furthermore, due to the strong polarization of the $\mathrm{C}-\mathrm{F}$ bond, $\mathrm{F} \cdot \mathrm{H}-\mathrm{C} \mathrm{H}$-bonds occur in organic fluorinated materials [23], which can affect in a significant way molecular geometry and the charge distribution of the molecules, playing an interesting role in the solid state organization. In light of these considerations, the fluoro-functionalization has been explored as a chemical and structural modification in several classes of organic materials. In this review, we describe our contribution in this field, through the synthesis and electro-optical investigation of arylenevinylene compounds for application in luminescent devices and photovoltaics.

\section{Results and Discussion}

\subsection{Fluorinated Oligo(arylenevinylene)s}

\subsubsection{Synthesis}

Although the initial interest for conjugated oligomers was related to their possible use as model compounds, aiming to investigate the properties of corresponding polymeric materials [24], very shortly, they themselves have become very attractive as active materials for electro-optical applications, showing, in some cases, even better performances with respect to their polydisperse homologs [25-29].

Recently, we have developed a versatile and stereoselective synthetic protocol to prepare all trans oligoarylenevinylenes fluoro-functionalized on the vinylene units, based on the Stille cross-coupling reaction. Additional polar and non-polar groups have been introduced onto aromatic rings in order to modulate the solubility of the final compounds in different solvents [30]. A series of oligomers (6-9) was prepared by reacting (E)-1-2-difluoro-2-[4-(octyloxy)phenyl]vinyl-1-tributilstannane 1 with diiodoaryls 2-5 in the presence of $\mathrm{Pd}\left(\mathrm{PPh}_{3}\right)_{4}$ as the catalyst, $\mathrm{CuI}$ in a stoichiometric amount, $\mathrm{DMF} / \mathrm{THF}$ as the reaction solvent, overnight at room temperature (Scheme 1). 
Scheme 1. Synthesis of fluorinated oligomers 6-9. a In situ hydrolysis by $40 \%$ aq KF.

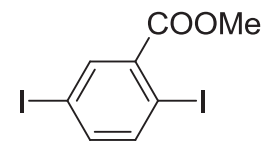

2
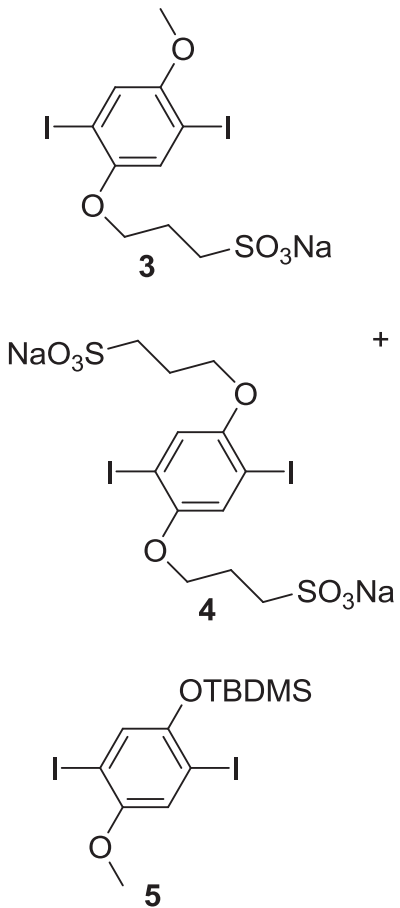
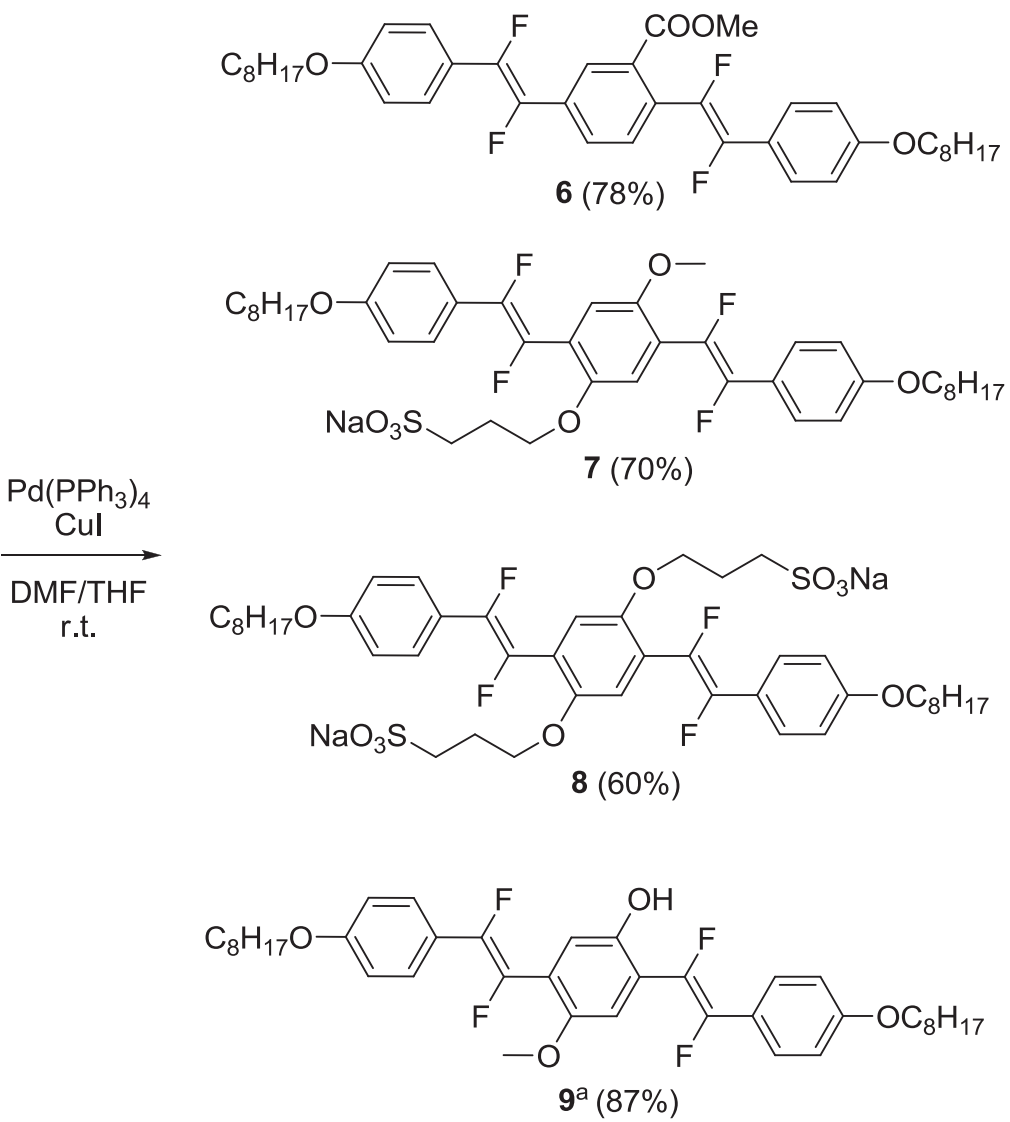

The Stille reaction proceeds regio- and stereo-selectively, affording oligoarylenevinylenes 6-9 with all-trans vinylene units. Compound 9 was obtained after in situ hydrolysis with $40 \%$ aq KF. Oligomers 6, 7 and 9 were purified by chromatography and obtained in good yields as a yellow solid, a dark orange solid and a pale yellow solid, respectively. Oligomer $\mathbf{8}$ was purified by several crystallizations from DMF/acetone and obtained pure as a grey solid. The alkoxy chains bonded to the terminal phenyl rings confer a good solubility to compounds 6, 7 and 9 in organic solvent, such as $\mathrm{CHCl}_{3}, \mathrm{CH}_{2} \mathrm{Cl}_{2}$, $\mathrm{MeOH}$, while compound $\mathbf{8}$ shows good solubility only in aprotic dipolar solvents, such as DMF and DMSO. Then, compound 6 was submitted to a basic hydrolysis with $\mathrm{LiOH} \cdot \mathrm{H}_{2} \mathrm{O}$ in $\mathrm{THF}, \mathrm{MeOH}$ and $\mathrm{H}_{2} \mathrm{O}$ to yield oligomer 10, bearing a free carboxylic group (Scheme 2).

Scheme 2. Synthesis of fluorinated oligomer 10.

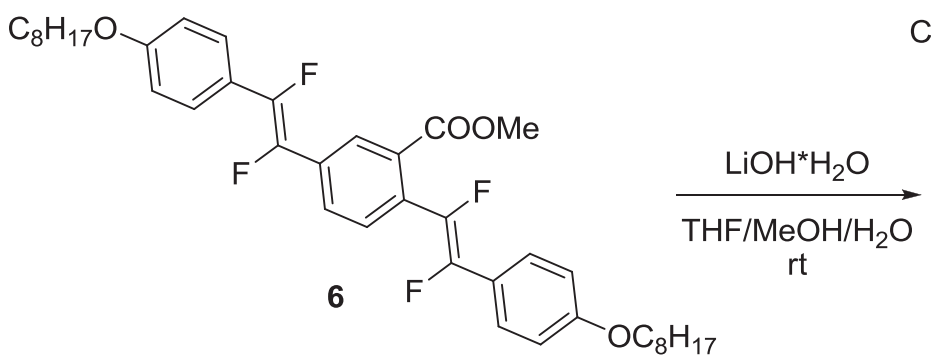<smiles>CCCCOc1ccc(/C(F)=C(\F)c2ccc(/C(F)=C(\F)c3ccc(OCCCC)cc3)c(C(=O)O)c2)cc1</smiles>

Compound 9 was converted in triflate derivative 11, which, in turn, reacted with commercial 4-(4,4,5,5-tetramethyl-1,3,2-dioxaborolan-2-yl)benzoate, 12, under the Suzuki reaction conditions, to 
yield intermediate 13. Finally, compound $\mathbf{1 3}$ was submitted to a basic hydrolysis, under the same conditions of 10, to yield oligomer 14 with the free carboxylic unit (Scheme 3).

Scheme 3. Synthesis of fluorinated oligomer 14.

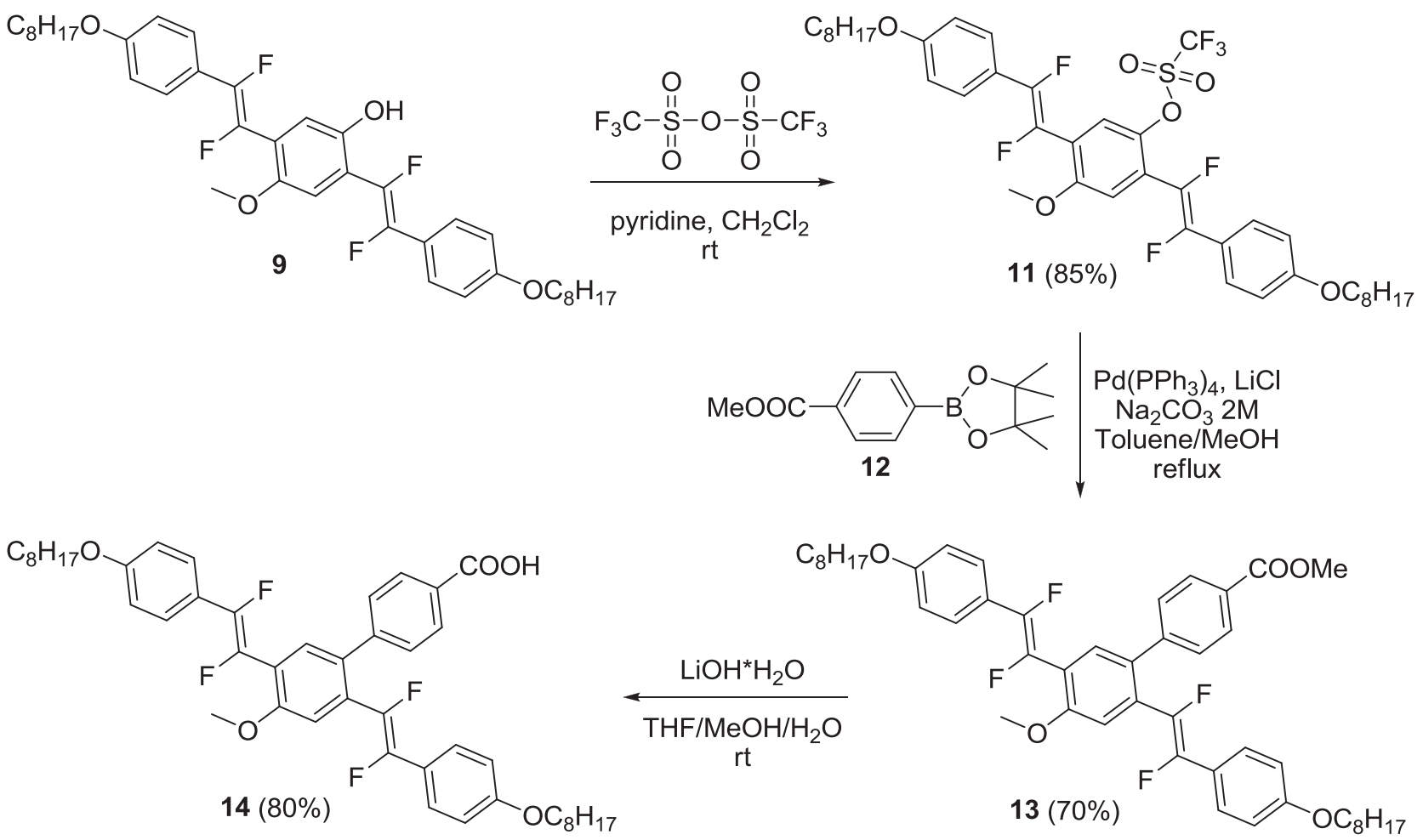

The synthesis of intermediate (E)-1,2-difluoro-2-[4-(octyloxy)phenyl]vinyl-1-tributilstannane 1 was carried out by extending a literature procedure [31], as shown in Scheme 4.

Scheme 4. Synthesis of organostannane 1.

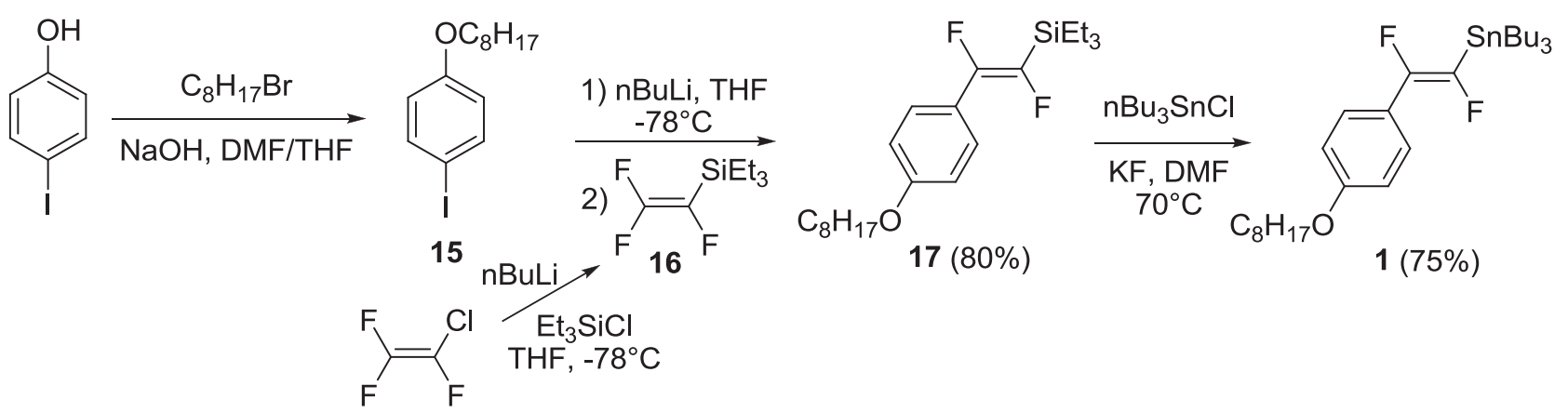

1-Iodo-4-(octyloxy)benzene 15 (obtained by alkylation of commercial 4-iodophenol) was lithiated by $\mathrm{nBuLi}$, then reacted with triethyl(trifluorovinyl)silane $\mathbf{1 6}$ (prepared by lithiation of commercial chlorotrifluoroethene with nBuLi followed by quenching with chlorotriethylsilane - see Scheme 7 and related discussion), to yield intermediate vinyl silane 17. This, in turn, was submitted to a transmetallation process with $\mathrm{nBu}_{3} \mathrm{SnCl}$ in the presence of anhydrous $\mathrm{KF}$ in dry $\mathrm{DMF}$ and at $70 \mathrm{C}$, to yield the organostannane $\mathbf{1}$. 
Fluorenevinylene oligomer (20) was also prepared following the same synthetic protocol adopted for the preparation of oligomers 6-9, starting from organostannane 18 (Scheme 5).

Scheme 5. Synthesis of fluorinated oligomer 20.

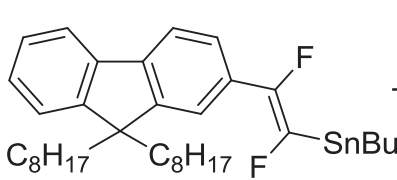

18

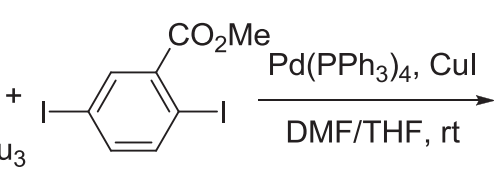

2

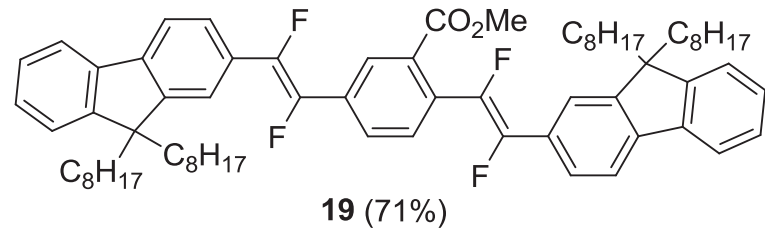

$19(71 \%)$
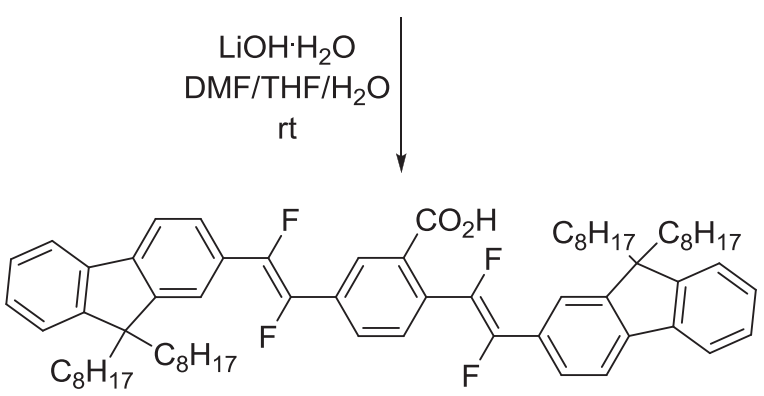

$20(92 \%)$

Organostannane 18 reacted with diiodoaryl 2 in the Stille reaction conditions, to yield intermediate compound 19. This was finally submitted to a basic hydrolysis with $\mathrm{LiOH} \cdot \mathrm{H}_{2} \mathrm{O}$ in THF, $\mathrm{MeOH}$ and $\mathrm{H}_{2} \mathrm{O}$, to yield the final oligomer, 20, with the free carboxylic unit. For the preparation of organostannane 18, a different synthetic pathway was followed with respect to organostannane $\mathbf{1}$, since the lithium derivative of 2-iodo-9,9-dioctylfluorene 21 (Scheme 6), obtained after its treatment with $\mathrm{nBuLi}$, did not react with triethyl(trifluorovinyl)silane 16. Therefore, intermediate 21 was coupled with (E)-[1,2-difluoro-(2-tributylstannyl)vinyl]triethylsilane 22 under the Stille reaction conditions, in the presence of $\mathrm{Pd}\left(\mathrm{PPh}_{3}\right)_{4}$ and $\mathrm{CuI}$ in $\mathrm{DMF} / \mathrm{THF}$, to yield compound 23 (Scheme 6).

Scheme 6. Synthesis of organostannane 18.

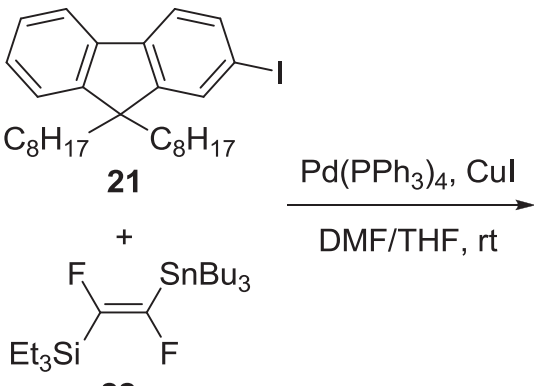

22

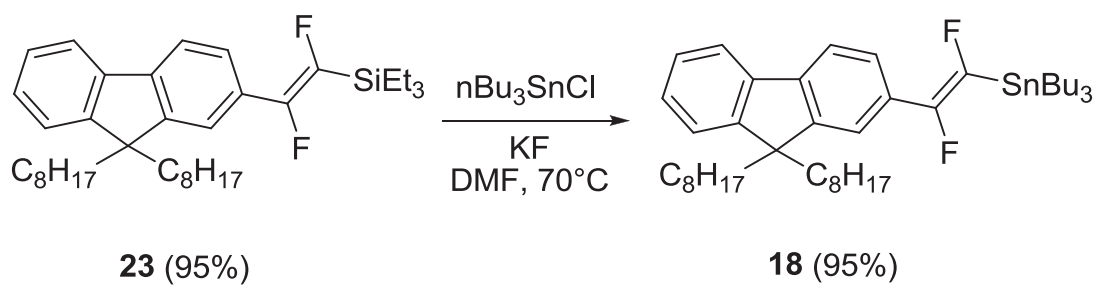

$18(95 \%)$

Vinylsilane 23 was then submitted to a transmetallation reaction with $\mathrm{nBu}_{3} \mathrm{SnCl}$ and anhydrous $\mathrm{KF}$ in dry DMF at $70 \mathbb{C}$, to yield organostannane $\mathbf{1 8 .}$

Compound 22, which can be used as a versatile organometallic building block in the preparation of asymmetrically 1,2-disubstitued difluorovinylene compounds, was synthesized for the first time in our laboratories following a synthetic protocol, which allows for preparation of two different 
organometallic reagents, (E)-[1,2-difluoro-(2-tributylstannyl)vinyl]triethylsilane $\mathbf{2 2}$ and (E)-1,2-difluoro-1,2-bis(tributylstannyl)ethene 26 [30] (Scheme 7).

Following a modified literature procedure [32], commercial chlorotrifluoroethene was lithiated by $\mathrm{nBuLi}$ and quenched with chlorotriethylsilane to yield intermediate 16. Compound $\mathbf{1 6}$ was selectively hydrogenated with $\mathrm{LiAlH}_{4}$ at $0 \mathrm{C}$ to yield difluorovinylsilane 24, which can be used as a starting reagent to synthesize compounds $\mathbf{2 2}$ or $\mathbf{2 6}$. The use of chlorotriethylsilane instead of chlorotrimethylsilane was introduced in order to increase the boiling point of intermediate compounds, 16 and 24, which can be, thus, easily separated from the reaction solvent by distillation. The same purification was not possible when chlorotrimethylsilane was used, because the trimethylsilyl derivatives corresponding to $\mathbf{1 6}$ and $\mathbf{2 4}$ have low boiling points very close to that of the reaction solvent. Treatment of intermediate 24 with $\mathrm{nBuLi}$ and $\mathrm{nBu}_{3} \mathrm{SnCl}$ afforded the organometallic compound, 22. Treatment of intermediate $\mathbf{2 4}$ with anhydrous $\mathrm{KF}$ and $\mathrm{nBu}_{3} \mathrm{SnCl}$ in dry $\mathrm{DMF}$ at $70 \mathfrak{C}$, resulted in a transmetallation process to yield difluorovinyltributylstannane $\mathbf{2 5}$. This was successively lithiated with lithium 2,2,6,6-tetramethylpiperide (obtained treating 2,2,6,6-tetramethylpiperidine with $\mathrm{nBuLi}$ ) and quenched with $\mathrm{nBu}_{3} \mathrm{SnCl}$, to yield bis-stannane 26. All intermediate compounds and final products in Scheme 7 are easily purified by distillation.

Scheme 7. Synthesis of intermediates 22 and 26.
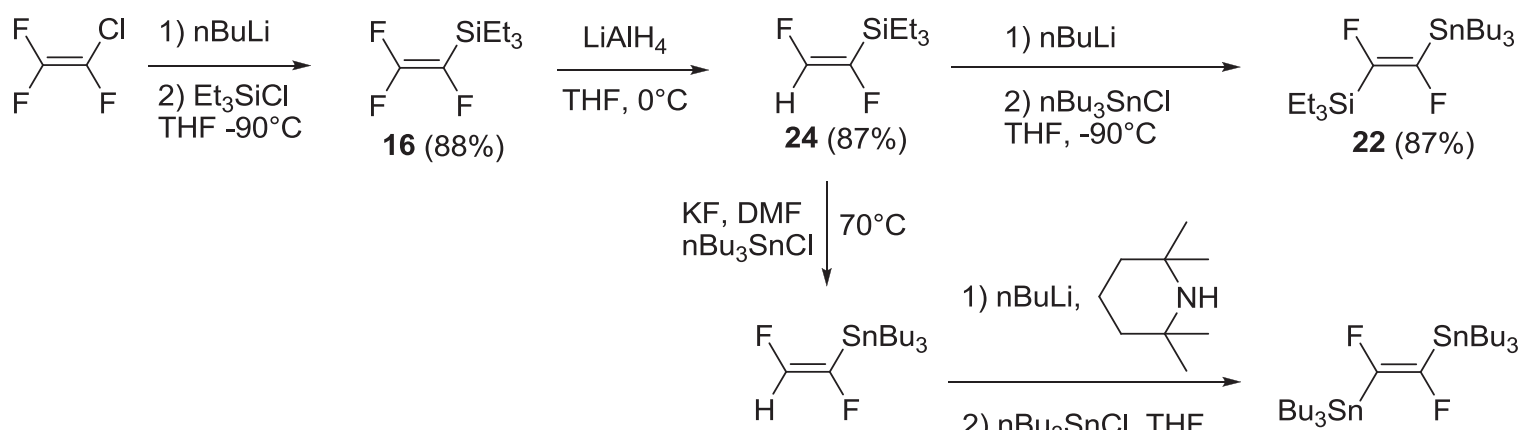

$25(75 \%)$
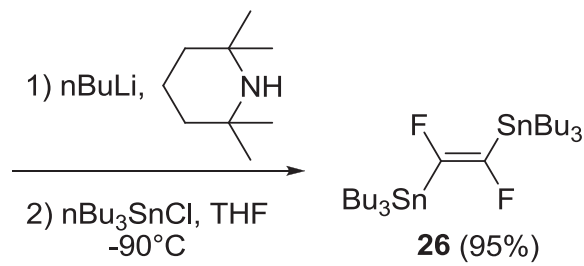

Taking advantage from organostannane 25, we set up a synthetic sequence to prepare compound MEH-OPDFV, the oligomer model of MEH-PPDFV, as reported in Scheme 8.

Compound 27 was selectively iodinated in the ortho-position with respect to the methoxy group by iodine, iodic acid and sulfuric acid in a mixture of chloroform, acetic acid and water as the solvent, to yield compound 28 . Then, the tosylate group was removed by $20 \%$ aq sodium hydroxide in t-BuOH, giving compound 29. Compound 29 reacted with 1-bromo-2-ethylhexane in the presence of $\mathrm{KOH}$ in DMSO to yield intermediate 30. Compound 30 reacted, in the Stille conditions, with organostannane 25 to yield intermediate 31. That was, in turn, converted in organostannane intermediate 32 by treatment with lithium 2,2,6,6-tetramethylpiperidide (prepared in situ) followed by quenching with $\mathrm{nBu}_{3} \mathrm{SnCl}$. Finally, intermediate $\mathbf{3 2}$ was coupled in the Stille reaction with diiodo derivative $\mathbf{3 3}$, to yield oligomer MEH-OPDFV, which was purified by chromatography and obtained as a colorless dense liquid. 
Scheme 8. Synthesis of fluorinated oligomer MEH-OPDFV.

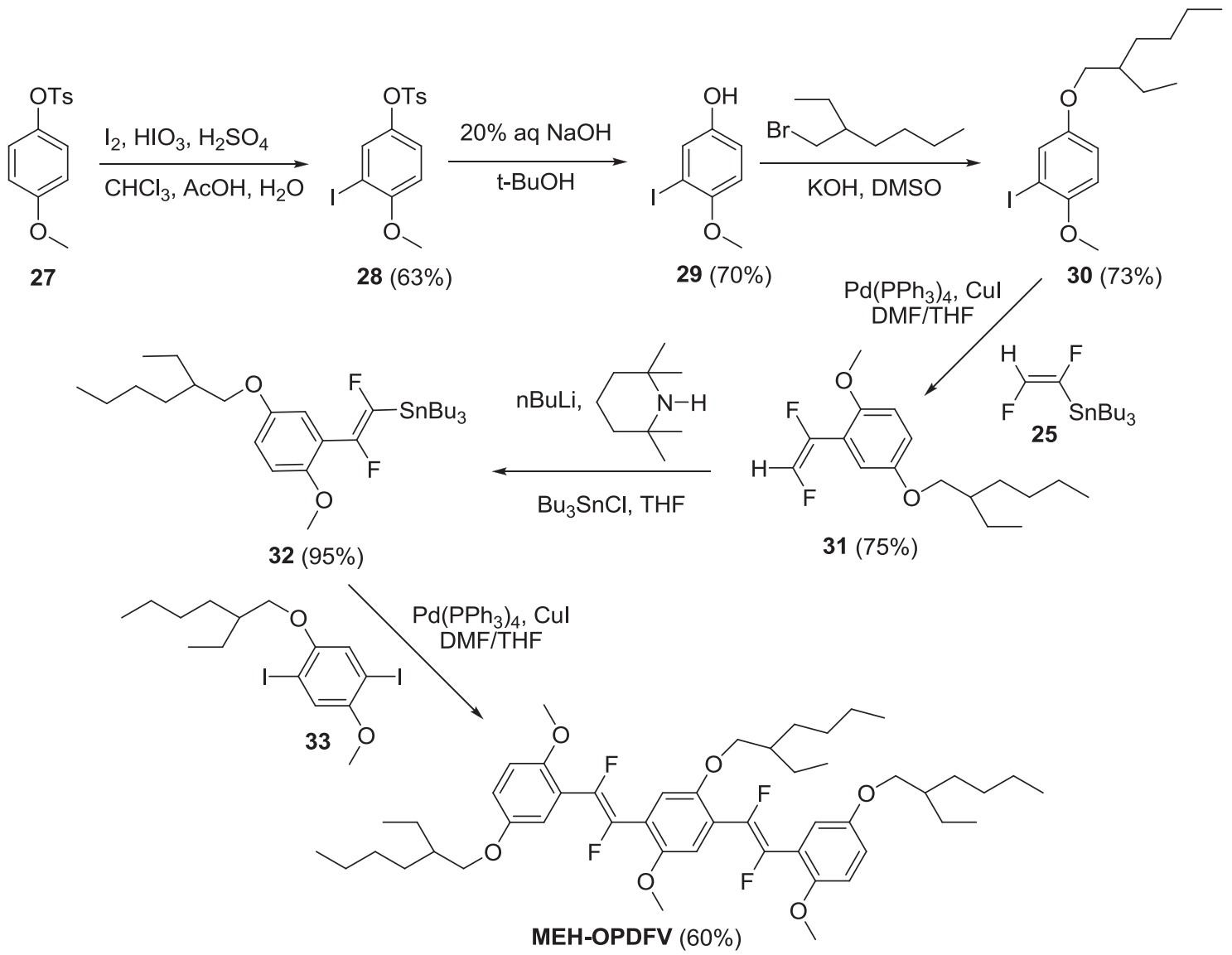

2.1.2. Spectroscopic Properties of Oligo(arylenedifluorovinylene)s

UV-Vis spectra of the synthesized oligomers are shown in Figure 1.

Figure 1. Absorption spectra of the oligomers in aerated solvents: 7, 14 and 20 were measured in $\mathrm{CH}_{2} \mathrm{Cl}_{2}, \mathbf{8}$ in DMSO and 10 in $\mathrm{MeOH}$.

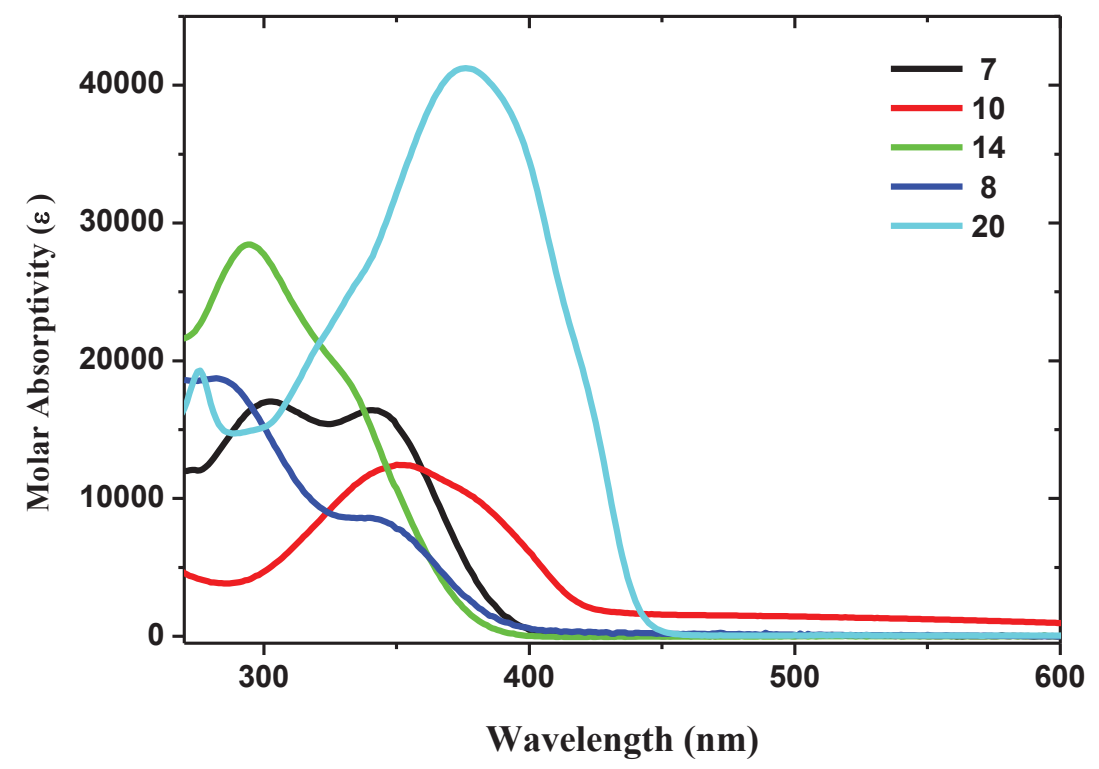


All oligomers show absorption peaks in the range 270-400 nm. Oligomer 20 shows the highest molar absorptivity coefficient, $\varepsilon$, with a spectrum shifted at a longer wavelength with respect to the others oligomers. The absorption peaks are assigned to $\pi-\pi^{*}$ transitions along the conjugated backbone, and the $\varepsilon$ values at $\lambda_{\max }$ range from about 14,000 to $20,000 \mathrm{M}^{-1} \mathrm{~cm}^{-1}$ for $\mathbf{7 ,} \mathbf{8}$ and $\mathbf{1 0}$, reaching about $30,000 \mathrm{M}^{-1} \mathrm{~cm}^{-1}$ for 14 and $40,000 \mathrm{M}^{-1} \mathrm{~cm}^{-1}$ for $\mathbf{2 0}$ (Table 1).

Table 1. Photophysical properties of oligomers. Emission quantum yields $\Phi$ in solution were measured using quinine sulfate in $0.5 \mathrm{M} \mathrm{H}_{2} \mathrm{SO}_{4}$ as the standard [33].

\begin{tabular}{cccc}
\hline Oligomer & $\begin{array}{c}\text { Molar Absorptivity } \\
\boldsymbol{\varepsilon}\left(\times \mathbf{1 0}^{\mathbf{3}} \mathbf{M}^{-\mathbf{1}} \mathbf{c m}^{-\mathbf{1}}\right)(\boldsymbol{\lambda}, \mathbf{n m})\end{array}$ & $\begin{array}{c}\text { Emission } \\
\boldsymbol{\lambda}_{\max }(\mathbf{n m})\end{array}$ & $\begin{array}{c}\boldsymbol{\Phi}(\mathbf{\%}) \\
(\text { solvent) }\end{array}$ \\
\hline 7 & $13(302), 12(342)$ & 436 & $0.87\left(\mathrm{CH}_{2} \mathrm{Cl}_{2}\right)$ \\
8 & $14(283), 6(342)$ & 440 & $0.97(\mathrm{DMSO})$ \\
10 & $14(352)$ & 466 & $5.4(\mathrm{MeOH})$ \\
14 & $29(295)$ & 458 & $0.43\left(\mathrm{CH}_{2} \mathrm{Cl}_{2}\right)$ \\
20 & $19(276), 41(376)$ & 444,471 & $8.12\left(\mathrm{CH}_{2} \mathrm{Cl}_{2}\right)$ \\
\hline
\end{tabular}

All oligomers are luminescent in solution with maxima peaks in the blue and blue-green region of the visible spectrum (Table 1, Figure 2). Oligomers 10 and 20 show the higher emission quantum yields. Oligomers 7 and 8 show similar emission profiles ( $\lambda_{\max }$ at 436 and $440 \mathrm{~nm}$, respectively), they are slightly blue shifted with respect to those of 10,14 and 20. These experimental observations are in accordance with theoretical calculations that assign the blue-shift to the torsional distortion of conjugated backbone induced by repulsive intramolecular interactions between fluorine atoms on the vinylene units and oxygen atoms of the alkoxy groups in the ortho-position on the aromatic rings [34].

Figure 2. Normalized emission spectra of the oligomers in aerated solvents: 7, 14 and 20 were measured in $\mathrm{CH}_{2} \mathrm{Cl}_{2}, \mathbf{8}$ in DMSO and $\mathbf{1 0}$ in $\mathrm{MeOH}$.

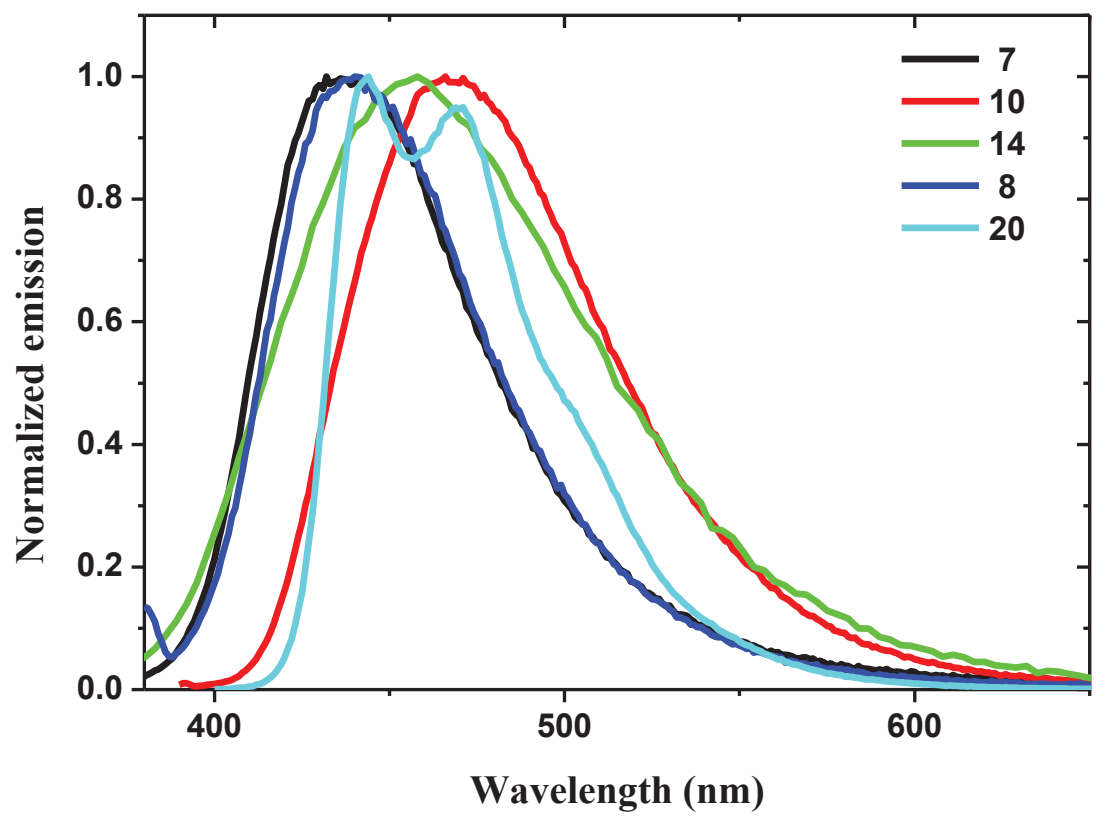


Oligomers 7, 8, 10 and 14 have the same main conjugated backbone, the only difference being in the functional groups bonded to the central aromatic unit. In oligomers $\mathbf{7}$ and $\mathbf{8}$, two alkoxy chains are bonded to the central phenyl unit. In oligomer 14, one of the two alkoxy groups is substituted by a phenyl unit bearing an electron-withdrawing carboxylic group, causing a broadening and a red shift $(458 \mathrm{~nm})$ of the emission. By substituting both alkoxy groups of the central phenyl unit with a single electron-withdrawing carboxylic group in oligomer 10, a further red shift (466 nm) in the emission is observed. Finally, oligomer 20, consisting of two fluorenedifluorovinylene units bonded to a central phenyl ring bearing a carboxylic group, shows two emission peaks at 444 and $471 \mathrm{~nm}$ and a shoulder at $500 \mathrm{~nm}$, with a typical profile of fluorene-based materials.

\subsubsection{Application of Oligomer 7 in a Chemical Sensor for Herbicide}

The arylenevinylene structure, containing fluorinated double bonds, alkoxy end chains and a propyloxy sulfonate group, confers to oligomer 7 an amphiphilic behavior that is an essential prerequisite for Langmuir Schaefer (LS) transfer. For this reason, it was chosen as the conjugated anionic counterpart of positively charged hydrophilic anthocyanins in the deposition of thin films using the LS approach [35]. Anthocyanins are well-known $\mathrm{pH}$ indicators, with the absorption maximum that shifts from red in acid environment towards blue at basic $\mathrm{pH}$, as a result of chemical structure variation [36]. Unfortunately, low stability prevents the use of anthocyanins pigments as active matrix in electronic devices, such as biosensors or dye sensitize solar cells. A number of physical and environmental factors affect anthocyanin stability, including temperature (the degradation rate increases with rising temperatures), UV light and $\mathrm{pH}$. In general, low $\mathrm{pH}$ values stabilize anthocyanins, while basic $\mathrm{pH}$ values can cause a rapid degradation. The hydrophilic nature of anthocyanins prevent their transfer by the LS method, even though the positive charge on the anthocyanins allows a supramolecular assembling with negatively charged species in a dyad system suitable for LS technique. Taking oligomer 7 as the anionic counterpart of anthocyanins (from Vitis vinifera), the electrostatic interaction between the two compounds has been exploited to obtain a stabile supramolecular dyad for film fabrication with the LS method. Experimental results confirm, in fact, that the strong electrostatic interaction existing between anthocyanins and oligomer 7 is able to prevent the aggregation of the former compound in water medium and stabilizes the LS film. In addition, LS films of the dyad oligomer 7/anthocyanins showed, also, an interesting increased stability of anthocyanins to UV exposure, with oligomer $\mathbf{7}$ acting as a protecting screen towards the UV radiation. Then, LS films of the oligomer 7/anthocyanins dyad were investigated as active matrix for herbicide sensors (Figure 3).

Absorption spectra of the LS film oligomer 7/anthocyanins deposited on a quartz substrate and incorporated in a home-made flow cell were acquired: the results showed a red shift and an almost complete bleaching of the absorption peak after exposition to hormonic herbicide, and the sensing process was completely reversible, disclosing the composite film as a potential colorimetric chemical sensor for the detection of a commercial herbicide. 
Figure 3. Variation of the visible spectrum of the LS sample, oligomer 7/anthocyanins, during exposure to a solution of a commercial hormone type pesticide (Erbitox E30). Reproduced with permission from [35]. Copyright 2012 Elsevier.

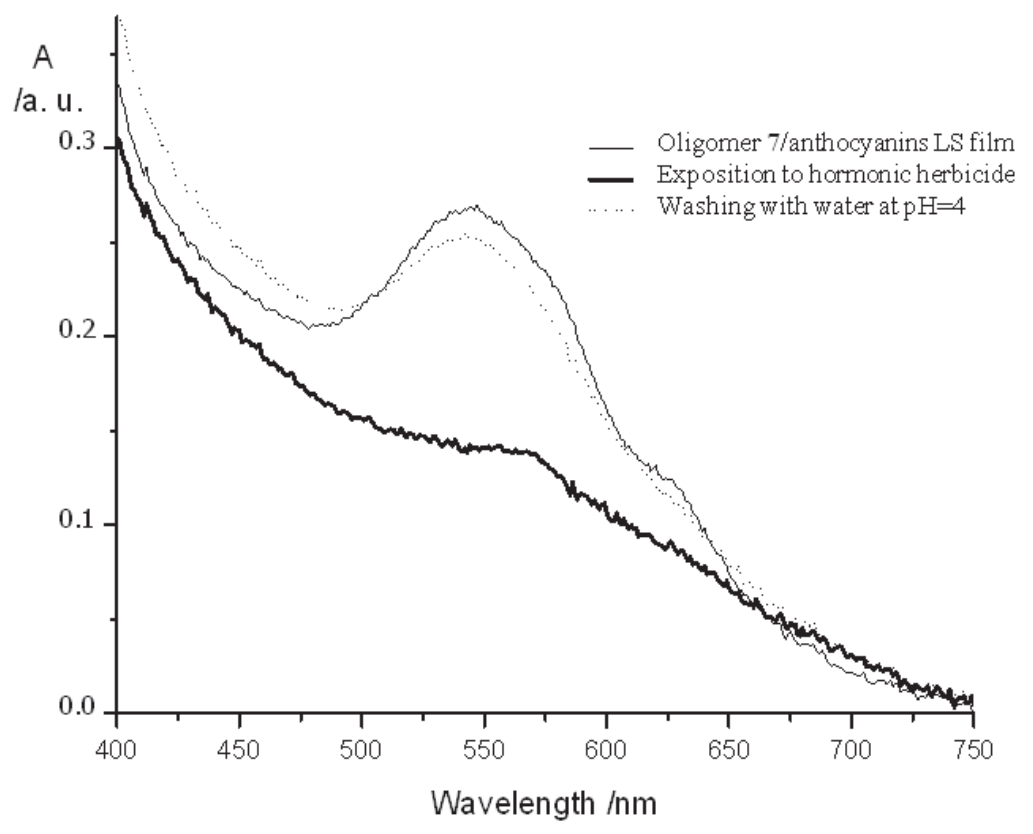

\subsection{Fluorinated Poly(arylenevinylene)s}

\subsubsection{Synthesis of Poly(arylenevinylene)s Fluorinated on the Aromatic Ring}

Polyarylenevinylenes (PAVs) represent one of the most interesting and investigated classes of conjugated polymers for applications in optoelectronics devices, since Friend and coworkers demonstrated their use as emitting materials in organic polymer-based light emitting diodes [37]. A number of synthetic methods have been developed to prepare a wide variety of polyarylenevinylene derivatives showing excellent performance in several optoelectronic applications. Among these, the - pecursor routes" (Gilch [38] or Wessling [39]) are versatile protocols and can be scaled up to a large scale production. They are based on the synthesis of soluble non-conjugated precursors that are converted in the final conjugated polymers after the elimination processes. Other classical methods are based on the Wittig [40-42] reaction and Knoevenagel [43-46] condensation. However, these methods do not allow accurate control of the stereochemistry of the vinylene units, which is of fundamental importance from the perspective of tailor electrical and optical properties of these materials for specific applications. Organometallic methods (i.e., Heck, Suzuki-Miyaura, Stille) are very attractive from this point of view, thanks to their intrinsic high regio- and stereo-selectivity, which permits a large number of conjugated arylenevinylene materials to be prepared, both oligomers and polymers, with a high degree of structural stereo- and regio-regularity. In particular, the Stille reaction is highly stereoselective and extremely versatile in the synthesis of PAVs, starting from (E)-1,2-bis(tributylstannyl)ethene 34, an organostannane building block that can be prepared in a large amount as a trans-vinylene unit source, used in cross-coupling with a number of aryl bis-halides. We have widely exploited the Stille reaction as a general synthetic strategy to prepare PAVs with various functional groups [47-50], with special attention, in the framework of this review, to the fluoro-functionalization, both on the aromatic ring 
and/or the vinylene units. Our investigation started with the synthesis of a PPV fully fluorinated on the aromatic rings, namely poly(tetrafluorophenylenevinylene) PTFPV. Before our work, unsuccessful attempts to synthesize the same polymer via a soluble precursor route [51] and via a self-condensation of (E)-2-(pentafluoropheny) ethenyl lithium [52] were reported by Brooke and Mawson. In our work, the Stille reaction was successfully carried out by coupling (E)-1,2-bis(tributylstannyl)ethene $\mathbf{3 4}$ and 1,4-diiodo-2,3,5,6-tetrafluorobenzene 35, in the presence of $\mathrm{Pd}\left(\mathrm{AsPh}_{3}\right)_{4}$ as the catalyst (generated in situ from $\mathrm{Pd}_{2} \mathrm{dba}_{3}$ and $\mathrm{AsPh}_{3}$ ) in benzene (Scheme 9) [53].

Scheme 9. Synthesis of PTFPV.

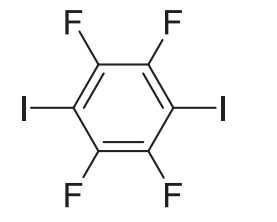

35

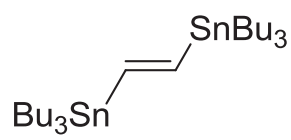

34

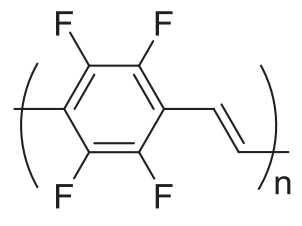

PTFPV $(95 \%)$

The usual $\mathrm{Pd}\left(\mathrm{PPh}_{3}\right)_{4}$ resulted in being ineffective as the catalyst of the cross-coupling between reagents 34 and 35, while substitution of the strong donor $\mathrm{PPh}_{3}$ ligand with the low donor $\mathrm{AsPh}_{3}$ ligand of palladium resulted in a very effective catalytic system. This experimental outcome is in according with data obtained in a previous study on the ligand effects in the Stille reaction [54]. In this study, the effect of changing the palladium ligands on the rates of a typical Stille cross-coupling (between iodobenzene and vinyltributyltin) was investigated, observing large rate enhancements associated with ligands of low donicity, like $\mathrm{AsPh}_{3}$ and tri(2-furyl)phosphine, compared to strong donors ligands, like the traditional $\mathrm{PPh}_{3}$. The pure polymer PTFPV was obtained in high yield as an insoluble yellow powder, after purification by washing the crude product of polymerization in a Soxhlet apparatus with hexane (24 h), methanol (24 h) and chloroform (12 h). It was characterized by Fourier transformed infrared spectroscopy (FTIR), X-ray photoelectron spectroscopy (XPS) and matrix assisted laser desorption ionization-time of flight (MALDI-TOF) mass spectrometry. Although the polymer was insoluble in common organic solvents, we were able to record its mass spectrum with the MALDI-TOF technique, using a protocol previously described for insoluble polyamides [55], which we extended for the first time to an insoluble conjugated polymer. The mass spectrum revealed peaks with masses ranging from 500 to $4500 \mathrm{amu}$, with average molecular weights of $M \mathrm{w}=2400$ and $M n=1700$ and a polydispersity index of D $>1.4$ (Figure 4). From the XPS characterization, assuming statistical chain terminations with one iodine atom and one tributylstannyl group, an average polymerization degree of approximately 17-20 arylenevinylene units can be deduced. The slight difference between the two methods may be attributed to the underestimated real molecular weights from the MALDI-TOF analysis, due to the mass discrimination phenomena at the higher molecular weights, which is intrinsic in this technique. The MALDI-TOF spectrum allowed also the correlation of the recorded peaks to PTFPV chains with all the possible chain terminations (Figure 5). 
Figure 4. MALDI-TOF mass spectrum of PTFPV.

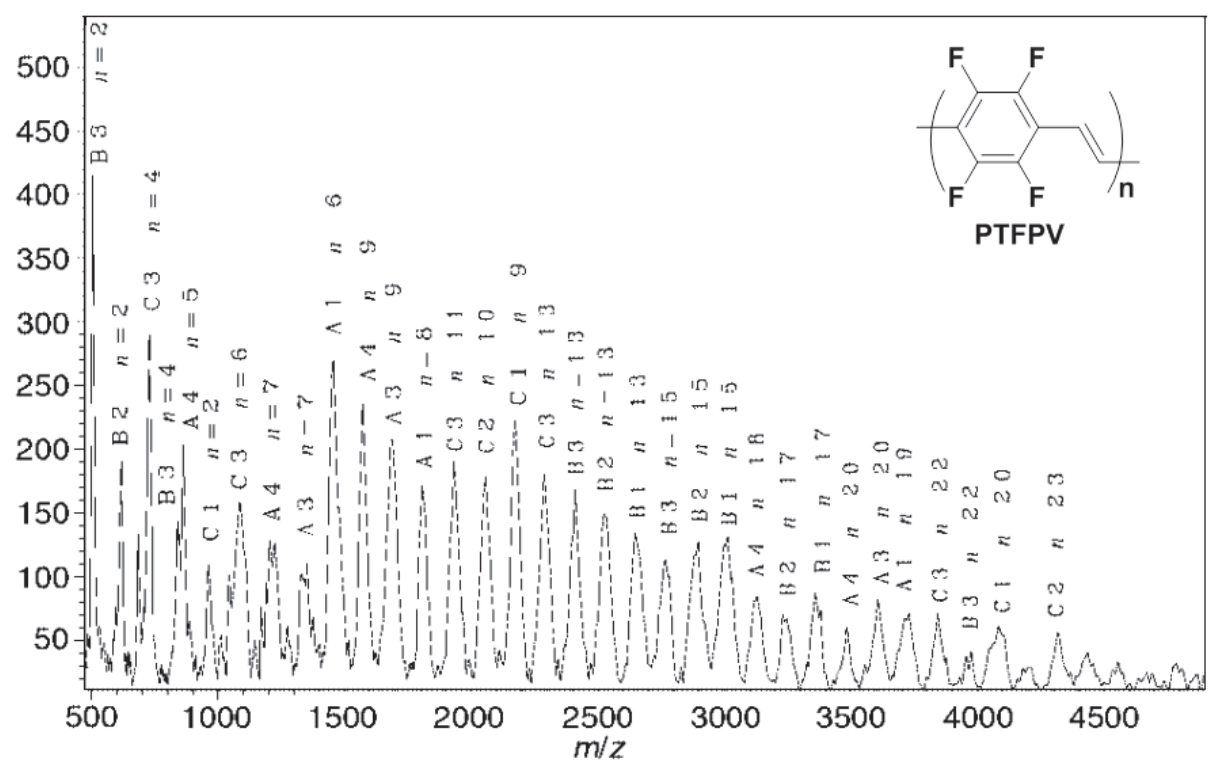

Figure 5. Polymeric chains of PTFPV detected in the MALDI-TOF mass spectrum.

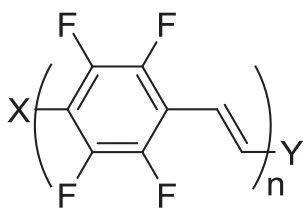

$\mathrm{A}_{1} \mathrm{X}=\mathrm{I} \quad \mathrm{Y}=\mathrm{SnBu}_{3}$

$\mathrm{A}_{2} \mathrm{X}=\mathrm{H} \mathrm{Y}=\mathrm{SnBu}_{3}$

$A_{3} X=I \quad Y=H$

$\mathrm{A}_{4} \mathrm{X}=\mathrm{HY}=\mathrm{H}$

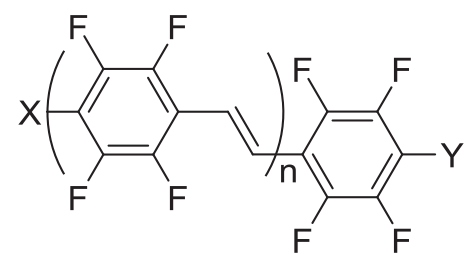

$\begin{array}{ll}B_{1} X=I & Y=1 \\ B & =1\end{array}$

$B_{2} X=H \quad Y=I$

$B_{3} X=H \quad Y=H$

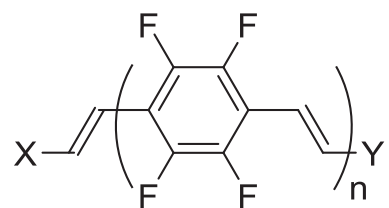

$\mathrm{C}_{1} \mathrm{X}=\mathrm{SnBu}_{3} \quad \mathrm{Y}=\mathrm{SnBu}_{3}$

$\mathrm{C}_{2} \mathrm{X}=\mathrm{H} \quad \mathrm{Y}=\mathrm{SnBu}_{3}$

$\mathrm{C}_{3} \mathrm{X}=\mathrm{I} \quad \mathrm{Y}=\mathrm{H}$

The FTIR confirmed the trans-configuration of vinylene units in the polymer by a characteristic strong band at $963 \mathrm{~cm}^{-1}$, due to the out of plane bending of the vinyl $\mathrm{C}-\mathrm{H}$ bonds with trans geometry.

It was demonstrated that the introduction onto the PPV skeleton of units with different electron affinity, such as alkoxy electron-donating and cyano electron-withdrawing groups, can be used as a valuable strategy to modify and adjust the HOMO and LUMO energy levels of the materials, obtaining a better matching with the work function of metal electrodes in OLED devices, thus improving performances [56,57]. In this contest, fluorine atoms appeared very interesting as for electron-withdrawing functionalities. Cacialli and coworkers [58] in 2000 reported the synthesis of random PPV copolymers, 36, containing fluorine and alkoxy substituent, through the Gilch protocol starting from bis-(halomethyl) benzenes, $\mathbf{3 7}$ and $\mathbf{3 8}$ (Scheme 10).

Varying the monomers' feed ratios, three copolymers incorporating different fluorinated unit percentages, i.e., $7 \%, 14 \%$ and 19\% (weight ratios), respectively, were obtained. The loss of solubility of polymer chains when a higher percentage of fluorinated units was introduced prevented the synthesis of copolymers with a higher content of fluorinated units. By applying the Stille protocol, we were able to synthesize analogous random copolymers co(TFPV-DOPV)s, reacting (E)-1,2-bis(tributylstannyl)ethene $\mathbf{3 4}$ with variable ratios of the two aromatic monomers, 1,4-diiodo-2,3,5,6-tetrafluorobenzene 35 and 1,4-diiodo-2,5-bis(octyloxy)benzene 39 (Scheme 11) [59-61]. 
Scheme 10. Synthesis of random copolymers 36 by Gilch-type polymerization.

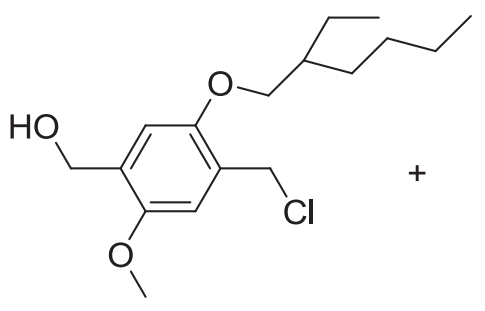

37

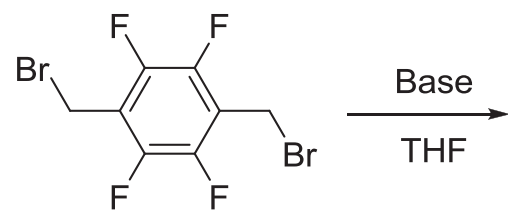

38

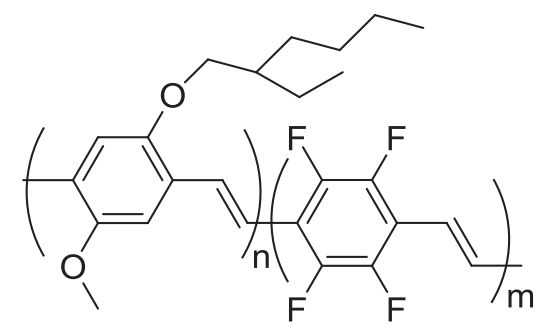

36

Scheme 11. Synthesis of random copolymers co(TFPV-DOPV)s by Stille cross-coupling.<smiles>Fc1c(F)c(I)c(F)c(F)c1I</smiles>

35<smiles>CCC[SbH2]C=C[SbH2]</smiles>

34

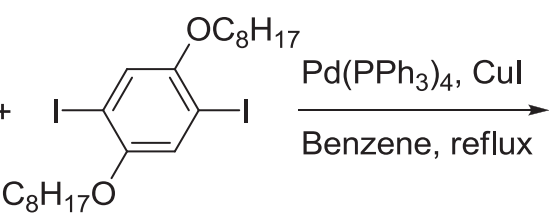

39

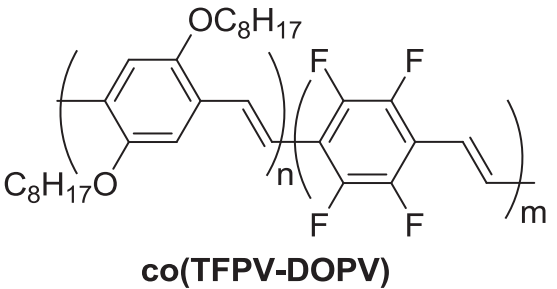

When an equimolar feed ratio of the two monomers, 35 and 39, was used, the polymerization reaction resulted in a random copolymer co(TFPV-DOPV) (soluble in chlorinated solvents, such as $\mathrm{CH}_{2} \mathrm{Cl}_{2}$ and $\mathrm{CHCl}_{3}$ ) with a percentage of tetrafluorophenylene units as high as $63 \%$, indicating a preferential incorporation of the fluorinated monomer (35), as a consequence of a higher reactivity of this reagent with respect to the alkoxy-substituted (39) one. The larger percentage of tetrafluorophenylene units in the random copolymer co(TFPV-DOPV), achievable with the Stille protocol with respect to the Gilch polymerization, can be attributed to a better solubility of the resulting polymers, due to the low molecular weight $(M \mathrm{n}=2200$ and $M \mathrm{w}=2500$, determined by MALDI-TOF mass analysis; $M \mathrm{n}=2200$ and $M \mathrm{w}=3400$, determined by size exclusion chromatography SEC).

\subsubsection{Optical and Electro-Optical Properties of Poly(arylenevinylene)s Fluorinated on the} Aromatic Ring

In Figure 6, the normalized absorption (a) and emission (b) spectra in thin film of the copolymer co(TFPV-DOPV) are compared with those of the two related homopolymers, the fluorinated PTFPV and the dioctyloxy substituted PDOPV. Films of PDOPV and co(TFPV-DOPV) were spin coated from chloroform solution, while the PTFPV film was thermally evaporated under reduced pressure $\left(10^{-6} \mathrm{~mm} \mathrm{Hg}\right)$. The optical energy gap ( $\left.\mathrm{Eg}\right)$ estimated from the absorption spectra are, respectively, $2.95 \mathrm{eV}$ for PTFPV, $2.32 \mathrm{eV}$ for PDOPV and $2.51 \mathrm{eV}$ for co(TFPV-DOPV). The polymer PDOPV shows a maximum peak (HOMO-LUMO transition) at $\lambda_{\max }=460 \mathrm{~nm}$, while PTFPV shows a maximum peak at $\lambda_{\max }=350 \mathrm{~nm}$. The strong blue shift observed in the latter can be attributed to the inductive electron-withdrawing effect of the fluorine atoms on the aromatic rings $[14,62]$, which is opposite to the electron-donating effect of the alkoxy substituents of PDOPV. 
Figure 6. Normalized absorption (a) and emission (b) spectra of PTFPV, PDOPV and co(TFPV-DOPV) thin films.
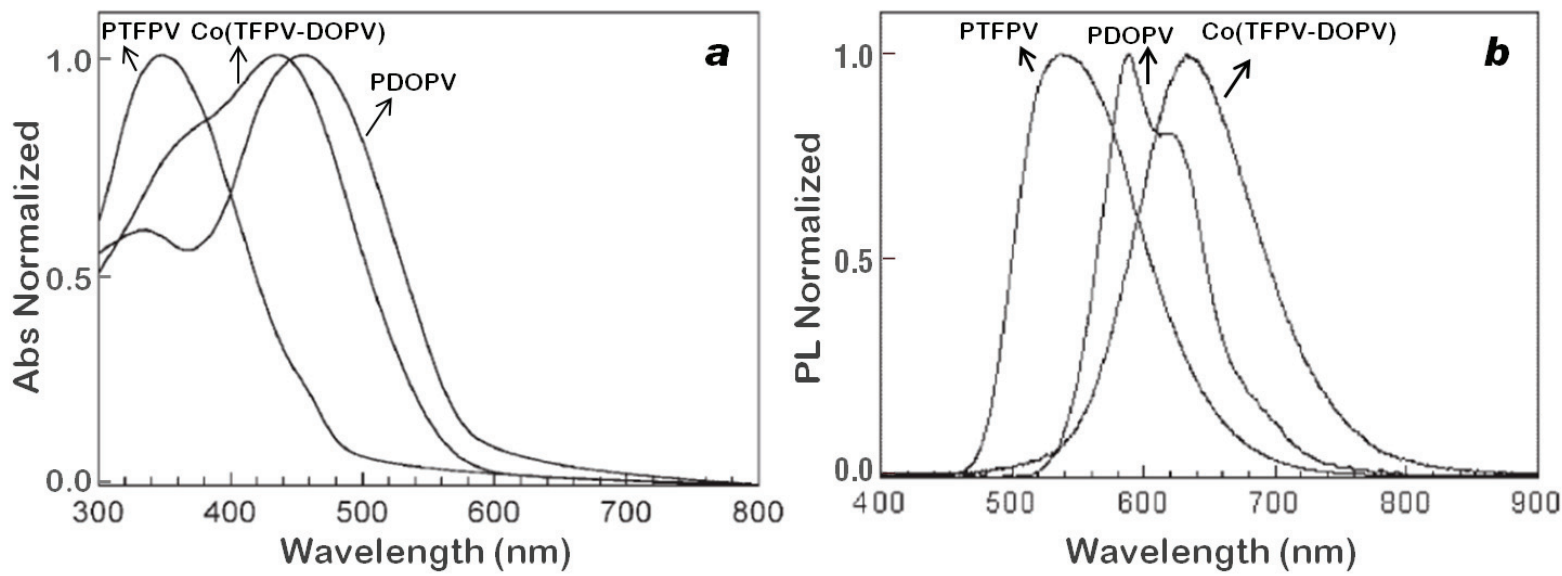

Figure 7. Emission spectra of co(TFPV-DOPV) in solution (dotted line) and thin film (solid line).

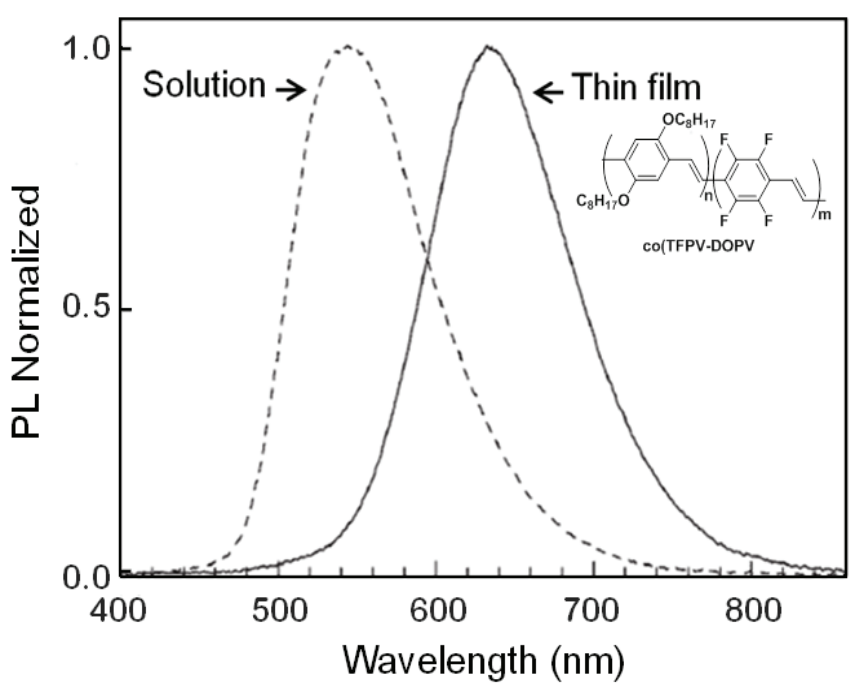

The copolymer, co(TFPV-DOPV), shows an absorption spectrum with two maxima at 440 and $360 \mathrm{~nm}$, which are close to the main absorption peaks of the two related homopolymers, thus suggesting a conjugated backbone containing two differently substituted polyphenylenevinylene segments, randomly arranged in the copolymer. The peak at $440 \mathrm{~nm}$ originates from chain segments containing the alkoxy substituted monomers, and it is slightly blue-shifted compared to the transition observed in pristine PDOPV, due to the presence of the electron-poor PTFPV segments. At the same time, the peak at $360 \mathrm{~nm}$ originates from PTFPV segments and is red-shifted with respect to the corresponding transition in pristine PTFPV, due to the presence of donor PDOPV segments. A different trend is observed in the emission spectra of the three polymers (Figure 6b); in fact, the emission maxima for PDOPV, PTFPV and co(TFPV-DOPV) are 580, 520 and $645 \mathrm{~nm}$, respectively, with a significant red shift for the copolymer with respect to the homopolymers. All three polymers show emission spectra red-shifted with respect to the corresponding absorption spectra. In particular, PDOPV and co(TFPV-DOPV) show Stokes' shifts of 110 and $190 \mathrm{~nm}$, respectively. The higher 
Stokes shift observed for co(TFPV-DOPV) can be attributed to the more effective formation of interchain excited species in the solid state, due to the simultaneous presence of electron rich DOPV and electron poor TFPV segments. This consideration is supported by the broader and structureless shape of the co(TFPV-DOPV) emission spectrum in thin film. Moreover, for co(TFPV-DOPV), the comparison of the emission spectra in solution and thin film (Figure 7) shows, as expected, a strong red-shift from solution to thin film, confirming the presence of exciton interchain migration, which is enhanced in the solid state.

Nonlinear optical properties (useful for applications in optical telecommunications) of co(TFPV-DOPV) and PDOPV have been investigated by measuring the third-order nonlinear susceptibility coefficient, $\chi^{(3)}$, in chloroform solution at $\lambda=1064 \mathrm{~nm}$, with the picoseconds Z-scan technique $[59,60,63]$. A considerable improvement of $\left|\chi^{(3)}\right|$, exceeding one order of magnitude, was observed for co(TFPV-DOPV) $\left(10^{10}\left|\chi^{(3)}\right| \sim 6 \mathrm{esu}\right)$ with respect to PDOPV $\left(10^{10}\left|\chi^{(3)}\right| \sim 0.5 \mathrm{esu}\right)$. Measurements in thin film have been also performed by the Third Harmonic Generation technique in the infrared range between 1.2 and $2.1 \mathrm{~mm}$, demonstrating an increased third order nonlinear optical coefficient of co(TFPV-DOPV) $\left(10^{11}\left|\chi^{(3)}\right| \sim 4.3 \mathrm{esu}\right)$ compared with those of the homopolymers PDOPV $\left(10^{11}\left|\chi^{(3)}\right| \sim 1.2\right.$ esu $)$ and PTFPV $\left(10^{11}\left|\chi^{(3)}\right| \sim 1.2\right.$ esu). The enhancement of the nonlinear optical coefficients in co(TFPV-DOPV) compared to the corresponding homopolymers, PDOPV and PTFPV, can be attributed to the simultaneous presence of electron rich and electron poor units in the polymeric chains, which increase the polarizability of the whole conjugated system.

Finally, electrochemical and photoinduced spectroelectrochemical studies have been performed on co(TFPV-DOPV), in order to investigate the nature of the species generated upon excitation and compare the photogenerated species with the electrochemical doping-induced charge carriers [64]. The result shows different infrared active vibration (IRAV) patterns for the electrochemical p- and n-doped co(TFPV-DOPV), indicating the existence of different structures of positive and negative charge carriers during the electrochemical doping. Moreover, the photoinduced absorption IRAV patterns were found similar to the IRAV band patterns obtained during the electrochemical reduction (n-doping) and differ from that of electrochemical oxidation (p-doping). Such a behavior, observed for the first time in a conjugated polymer, makes co(TFPV-DOPV) an electrochemically both p- and n-dopable material, which may be important for the construction of optoelectronic devices, where photogeneration of negative charge carriers is required.

The electroluminescent properties of the fluorinated polymer PTFPV have been investigated in an ITO/TPD/PTFPV/Al OLED configuration, where the PTFPV thin film was deposited by thermal evaporation under reduced pressure $\left(10^{-6} \mathrm{~mm} \mathrm{Hg}\right)$ [53]. The presence of fluorine atoms assists the electron injection from the Al cathode, but raises the energy barrier to the hole injection from the ITO anode. In fact, the addition of a hole transporting layer (TPD) between the anode and the electroluminescent PTFPV layer was necessary to obtain a working device, characterized by green light emission and an electroluminescent threshold voltage of $6.5 \mathrm{~V}$.

\subsubsection{Synthesis of Poly(arylenevinylene)s Fluorinated on the Vinylene Units}

Among the main drawbacks that limit commercial applications of PAVs in optoelectronic devices, the poor stability against the photodegradation processes, primarily localized on the double bonds, is 
certainly one of the most important. Overcoming this issue is necessary to increase the life-time of devices to match commercial requirements. To achieve this, substitution of $\mathrm{C}-\mathrm{H}$ bonds in the vinylene units with stronger and less reactive $\mathrm{C}-\mathrm{F}$ bonds can be used as a chemical modification of the conjugated backbone of PAVs able to increase their thermal chemical stability. In 2004, Su and coworkers $[65,66]$ first reported the synthesis of two PPV polymers with fluorinated vinylene units via the Gilch reaction (Scheme 12).

Scheme 12. Synthesis of PPVs with fluorinated double bonds via the Gilch reaction.

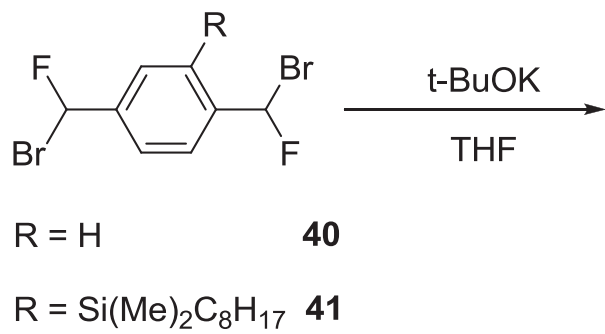

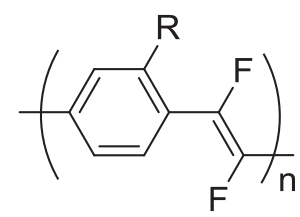

42

43

Moreover, the Gilch protocol requires the use of strong bases, which limits the generality of this approach to the synthesis of PPVs with functional group that are base-insensitive [67]. We investigated the possibility of extending the Stille protocol to the synthesis of PAV polymers fluorinated on the vinylene units, taking advantage of the availability of (E)-1,2-difluoro-1,2-ethenediyl)bis(tributylstannane) 26 (Scheme 7), an organometallic reagent that can be used as a convenient source of a difluorovinyl building block in cross-coupling reactions. Then, we successfully reacted reagent $\mathbf{2 6}$ with various diiodoaryl derivatives 44-47, as reported in Scheme 13 [68].

Scheme 13. Synthesis of PAVs with fluorinated double bonds via the Stille cross-coupling.

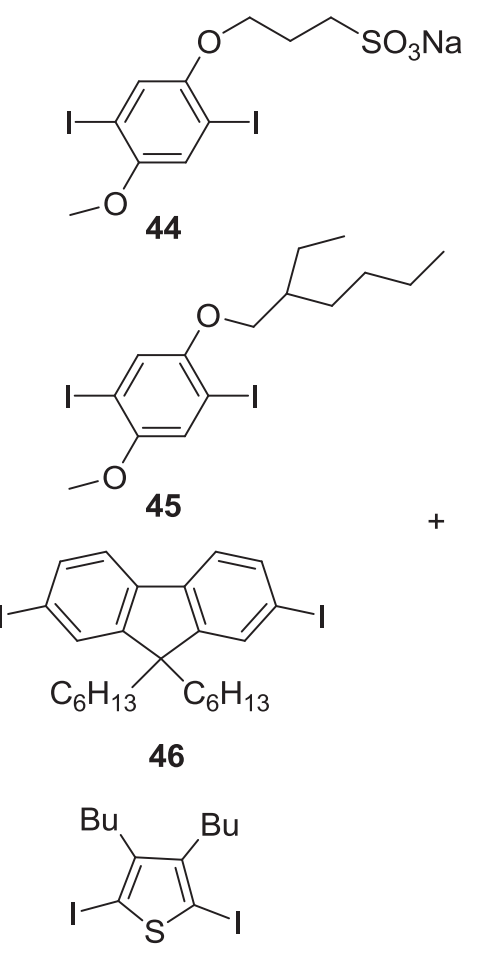

47
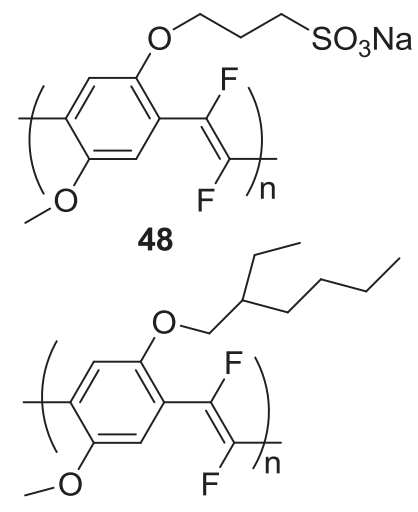

MEH-PPDFV

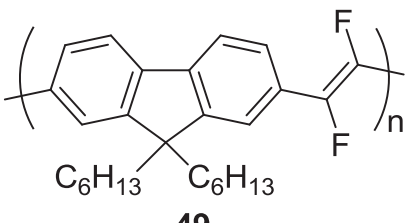

49

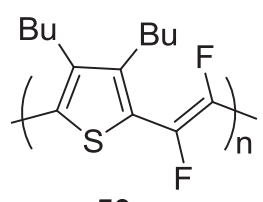


All polymerization was carried out at room temperature for one week, using $\mathrm{Pd}\left(\mathrm{PPh}_{3}\right)_{4}$ as the catalyst and $\mathrm{CuI}$ in a stoichiometric amount in DMF/THF. A final end-capping with phenyl tributyltin and iodobenzene is necessary for elimination of iodine and tributyltin terminal groups, respectively. Polymer 48 was purified by several crystallizations from DMF/acetone and obtained as a yellow-green powder. Polymers, MEH-PPDFV and 49, were purified by Soxhlet extraction with hexane (24 h), methanol $(24 \mathrm{~h})$ and chloroform $(12 \mathrm{~h})$. The pure products were recovered from chloroform and obtained as green and light-green powders, respectively. Polymer $\mathbf{5 0}$ was purified by extraction in a Soxhlet apparatus with dichloromethane, followed by crystallization from dichloromethane/methanol (twice) and obtained as a red powder. The average molecular weights $M \mathrm{n}$ and $M \mathrm{w}$ of polymers MEH-PPDFV, 49 and 50 were determined by conventional size exclusion chromatography (SEC), while a multiangle light scattering (MALS) detector on line to a SEC system was used for polymer 48 (Table 2).

Table 2. Yields, molecular weights and polydispersivities of polymers 48-50 and MEH-PPDFV.

\begin{tabular}{cccc}
\hline Polymer & Yield (\%) & Mw & Mw/Mn \\
\hline 48 & 55 & 65150 & 1.6 \\
MEH-PPDFV & 87 & 47000 & 1.7 \\
49 & 82 & 45600 & 2.0 \\
50 & 50 & 47100 & 2.9 \\
\hline
\end{tabular}

The Stille protocol was also extended to the synthesis of two new perfluorinated poly(arylenevinylene)s, namely poly(1,4-tetrafluorophenylenedifluorovinylene) 6F-PPV and poly(2,5-difluorothienylenedifluorovinylene) 4F-PTV, starting from organostannane 26 coupled with the diiodo perfluorinated aryl compounds 35 and 51, respectively (Scheme 14) [69].

Scheme 14. Synthesis of polymers 6F-PPV and 4F-PTV.<smiles>Fc1c(F)c(I)c(F)c(F)c1I</smiles>

35<smiles>Fc1c(I)sc(I)c1F</smiles>

51

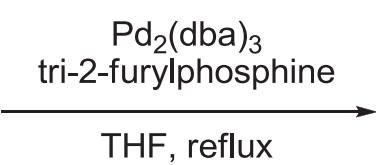<smiles>CCCCC(F)=C(F)[SbH2]C(C)(C)C</smiles>

26

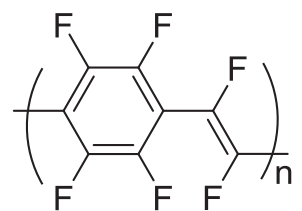

6F-PPV<smiles>CC(C)(C)/C(F)=C(\F)c1sc(C(C)(C)C)c(F)c1F</smiles>

4F-PTV

Polymer 4F-PTV was synthesized using the same catalytic system employed for the preparation of polymers 48-50 and MEH-PPDFV, while polymer 6F-PPV required a different catalyst, generated in situ from $\mathrm{Pd}_{2}(\mathrm{dba})_{3}$ and tri-2-furylphosphine, in refluxing THF. Both the polymers 6F-PPV and 4F-PTV were purified by washing the crude product of polymerization in a Soxhlet apparatus with hexane $(24 \mathrm{~h})$, methanol $(24 \mathrm{~h})$ and chloroform $(12 \mathrm{~h})$ and were collected as insoluble (in common 
organic and perfluorinated solvents) green and red powders, respectively. They were characterized by FT-IR and MALDI-TOF mass spectrometry. The IR spectra show two significant peaks due to the C-F stretching vibration of vinylene units $\left(1165 \mathrm{~cm}^{-1}\right.$ for 4 F-PTV and $1173 \mathrm{~cm}^{-1}$ for 6 F-PPV) and the C-F stretching vibration of aromatic rings $\left(1014 \mathrm{~cm}^{-1}\right.$ for $\mathbf{4 F - P T V}$ and $989 \mathrm{~cm}^{-1}$ for $\left.\mathbf{6 F - P P V}\right)$. The MALDI-TOF analysis reveals a polymerization length of 3-9 repeating units for 6F-PPV $(M \mathrm{n}=1330, M \mathrm{w}=1400$; polydispersity index, $\mathrm{D} \sim 1.05)$ and 6-12 for 4F-PTV $(M \mathrm{n}=1690$, $M \mathrm{w}=1750$; polydispersity index, $\mathrm{D} \sim 1.04$ ). Despite the mass discrimination phenomena in favor of low molecular weight chains, typical of the MALDI-TOF analysis, taking in account the low solubility of the growing polymer chains of these perfluorinated PAVs, we consider the molecular weights determined by MALDI-TOF mass spectra a good approximation of the real mass distribution of the final polymers. On the other hand, low molecular weight does not represent a limit to most applications of conjugated polymers as semiconductors, since also oligomers can exhibit optical and electrical properties suitable for device applications, with the additional advantage of using even the thermal evaporation-deposition as a processing method. This is, in fact, the case for polymers 6F-PPV and 4F-PTV, which can be thermally evaporated in good quality thin films under reduced pressure (about $10^{-5}$ mbar).

More recently, we focused our attention on the potentiality of fluorinated arylenevinylene polymers suitable for photovoltaic applications, as donor materials in bulk heterojunction (BHJ) solar cells. Some interesting papers [70-73] refer to favorable effects of the fluorine atoms on the performance of polymers used as donor materials in blend with phenyl-C61-butyric acid methyl ester (PCBM) derivatives in BHJ solar cells. The role of the fluorine is mainly attributed to its capability of lowering both the HOMO and LUMO energy levels of the donor, which causes an increase in the open circuit voltage, $\mathrm{V}_{\mathrm{OC}}$. Moreover, the increase in the thermal and chemical stability induced by the fluoro-functionalization of the organic materials makes this little and powerful substituent an intriguing tool to achieve organic-based long-lifetime devices. In search of new low band gap materials suitable for organic photovoltaics, the introduction of vinylene units in the conjugated backbone of polymers has been proposed as a structural modification to reduce the band gap, as in PPVs [74-83] and poly(thienylenevinylene)s (PTVs) [84-88]. In fact, several studies have reported on the effect of double bonds in donor polymers for BHJ solar cells, especially as a bridge between donor and acceptor units in donor-acceptor type low band gap polymers [89-98]. In our investigation, we focused our attention on the class of PAVs, with the main aim to study the effects of fluoro-functionalization of double bonds, in a molecular structure, which is characterized by a low band gap of the final polymer. Whit this aim, we designed and synthesized a PAV alternating fluorinated double bond and bis-thienyl-bis-alkoxy-benzothiadiazole units (PDTBTFV), together with its non-fluorinated counterpart (PDTBTV), starting from organostannane 26 and $\mathbf{3 4}$ coupled with diiodo derivative 52 in the Stille reaction conditions (Scheme 15) [99].

The polymers were purified by washing the crude product of polymerization in a Soxhlet apparatus with hexane $(24 \mathrm{~h})$ and methanol $(24 \mathrm{~h})$. A final extraction with chloroform yields the pure polymers as dark violet (PDTBTV) and dark blue (PDTBTFV) powder, in good yield. The average molecular weight was determined by size exclusion chromatography (SEC). The results are reported in Table 3. 
Scheme 15. Synthesis of polymers PDTBTFV and PDTBTV.

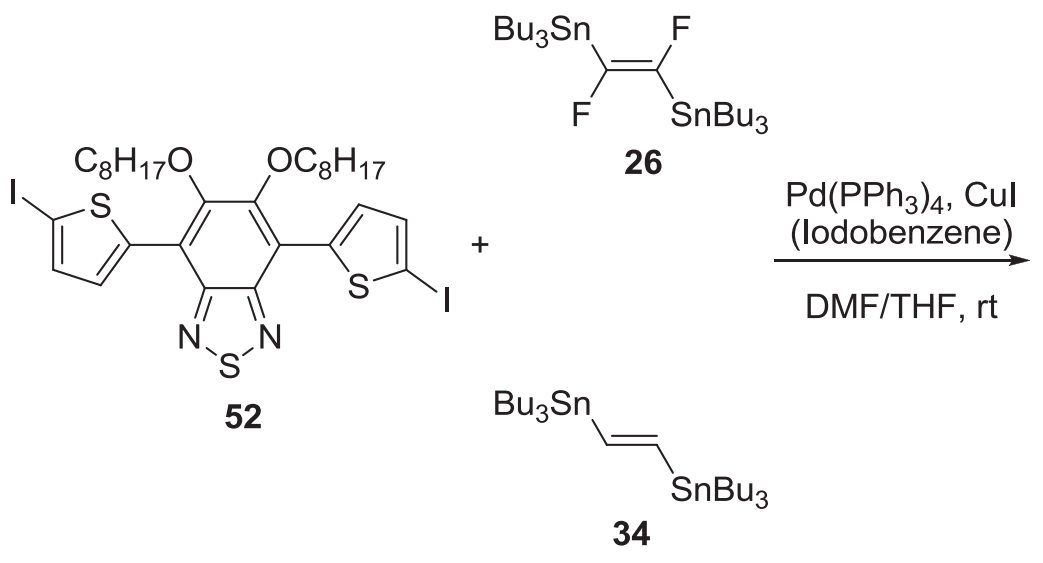

34

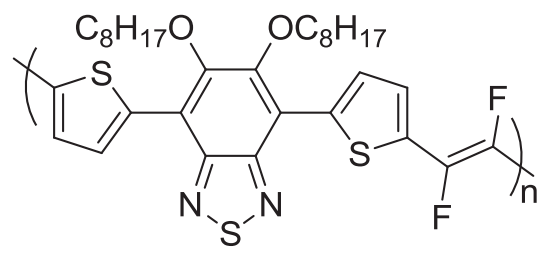

PDTBTFV

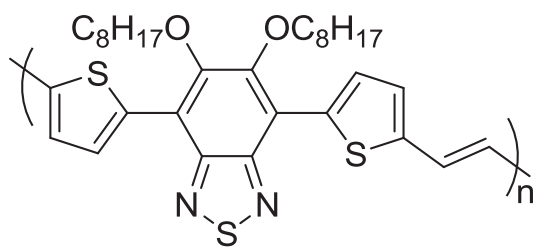

PDTBTV

Table 3. Yields, molecular weights and polydispersivities of polymers PDTBTV and PDTBTFV.

\begin{tabular}{cccc}
\hline Polymer & Yield (\%) & $\boldsymbol{M w}$ & $\boldsymbol{M w} / \boldsymbol{M n}$ \\
\hline PDTBTV & 70 & 36,900 & 4.67 \\
PDTBTFV & 75 & 43,700 & 4.46 \\
\hline
\end{tabular}

Both the polymers are soluble in chlorinated solvents, such as chloroform, chlorobenzene (CB) and ortho-dichlorobenzene (ODCB).

2.2.4. Optical and Electro-Optical Properties of Poly(arylenevinylene)s Fluorinated on the Vinylene Units

In order to study the effects of fluoro-functionalization of the double bond in alkoxy-substituted poly(arylenevinylene)s, we compared the spectroscopic properties of the fluorinated MEH-PPDFV with those of the non-fluorinated analogous, MEH-PPV. Fluorination of double bonds causes a strong hypsochromic shift of the absorption and emission spectra, both in solution and solid state. In fact, in chloroform solution, MEH-PPDFV showed a blue shift of $120 \mathrm{~nm}$ of the absorption maximum $\left(\lambda_{\max }{ }^{\text {abs }}=360 \mathrm{~nm}\right)$ with respect to MEH-PPV $\left(\lambda_{\max }{ }^{\text {abs }}=480 \mathrm{~nm}\right)$ and a corresponding blue shift of $87 \mathrm{~nm}$ was observed in the PL spectra $\left(\lambda_{\max }{ }^{\mathrm{PL}}=468 \mathrm{~nm}\right.$ for MEH-PPDFV and $\lambda_{\max }{ }^{\mathrm{PL}}=555 \mathrm{~nm}$ for MEH-PPV) (Figure 8).

Theoretical calculations on model oligomers (PVF-3, the fluorinated, and PVH-3, the non-fluorinated; Figure 9) performed by the Time Dependent Density Functional Theory (TD-DFT) [34] assign the large blue shift induced by the fluorination of the double bond mainly to the steric repulsion between the fluorine atoms on the vinylene units and the oxygen atoms of the alkoxy substituents on the neighboring phenylene units, rather than to the electron-withdrawing effect of the fluorine. The theoretical predictions were experimentally confirmed by the Raman spectroscopy measurements [100]. 
Figure 8. Normalized absorption (a) and emission (b) spectra in chloroform solution of MEH-PPDFV and MEH-PPV.
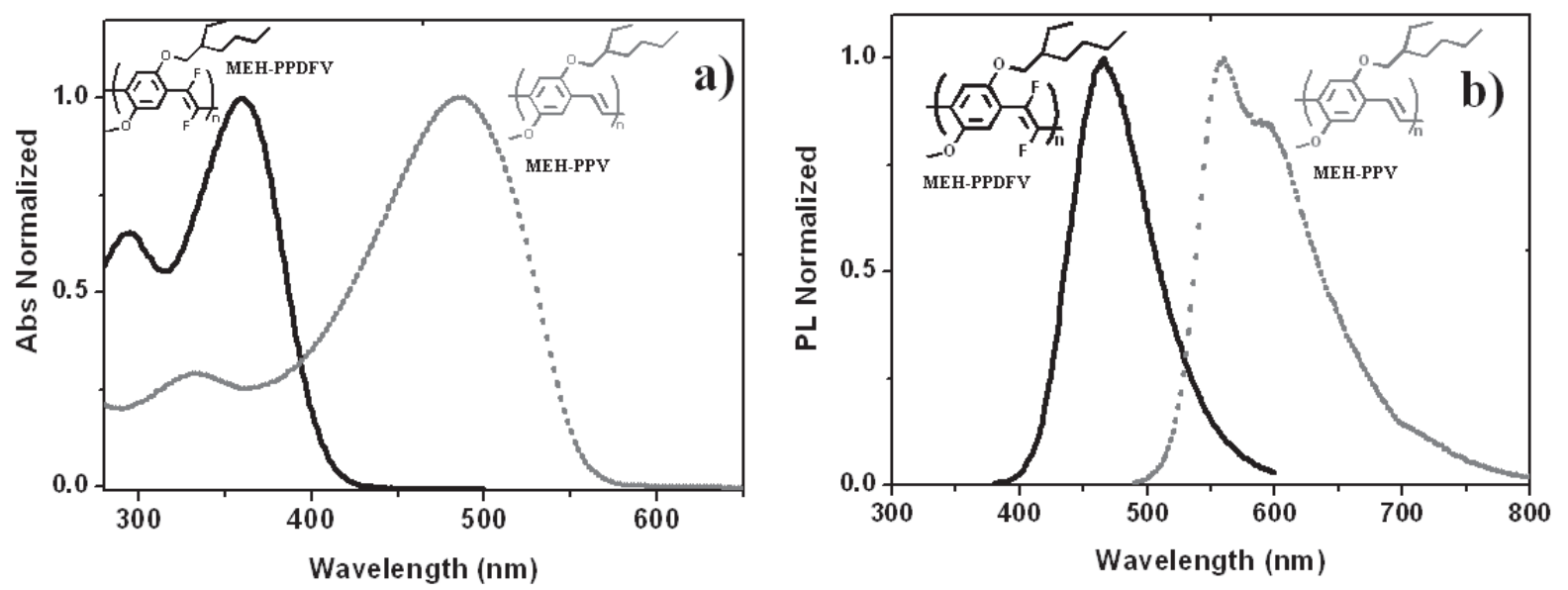

Through spectroscopic ellipsometry, the solid state optical properties of MEH-PPDFV and MEH-PPV have been investigated [101,102]. Thin films of MEH-PPDFV show an absorption maximum up to about $3.7 \mathrm{eV}(\sim 330 \mathrm{~nm})$, the highest value reported so far for a poly ( $p$-phenylenevinylene) polymer, and it is up to $30 \mathrm{~nm}$ blue shifted from the value measured for the same polymer in chloroform solution $\left(\lambda_{\max }{ }^{\text {abs }}=360 \mathrm{~nm}\right)$. Similarly, thin films of MEH-PPDFV show a strong blue photoluminescence in room temperature, with the emission maximum at $458 \mathrm{~nm}$, which is blue shifted of $10 \mathrm{~nm}$ with respect to the photoluminescence in solution $(468 \mathrm{~nm})$. This behavior is opposite to that observed for the polymer, MEH-PPV, and in general, for most conjugated polymers, where a red shift from solution to thin film, both for the emission and absorption peaks, is observed. The blue shift of the emission spectrum moving from solution to the solid state indicates that no interchain aggregation occurs for MEH-PPDFV in thin film. Moreover, in the solid state, the emission maximum of MEH-PPDFV is blue-shifted about $110 \mathrm{~nm}$ with respect to that of MEH-PPV $\left(\lambda_{\max }=568 \mathrm{~nm}\right)$, synthesized by the same methodology (Figure 10), resulting in the most blue shifted emission reported so far for a $\operatorname{poly}(p$-phenylenevinylene) polymer.

Figure 9. BH-LYP/TZVP optimized geometries of the model oligomers, PVF-3 and PVH-3. Reproduced with permission from [34]. Copyright 2008 the American Chemical Society.
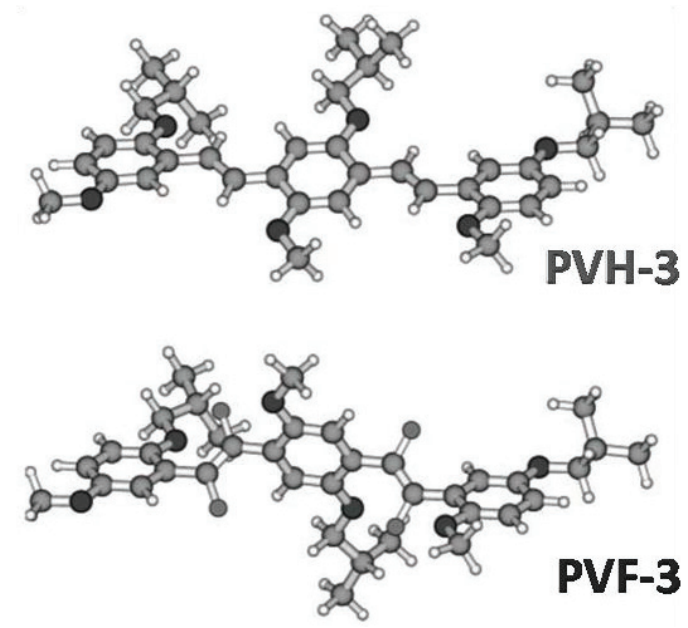
Figure 10. PL spectra of thin films of MEH-PPV and MEH-PPDFV.

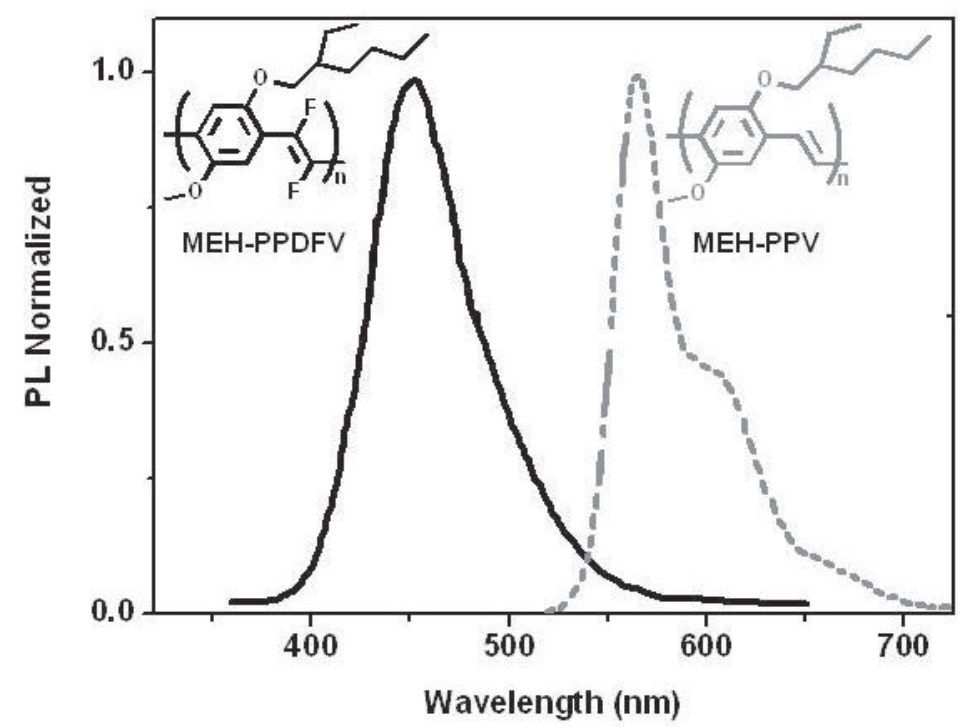

The electroluminescent properties of MEH-PPDFV have been investigated in comparison with those of MEH-PPV in a ITO/PEDOT:PSS/MEH-PPDFV(MEH-PPV)/Ba/Al OLED device (Figure 11 [103]). The EL spectra of MEH-PPDFV and MEH-PPV exhibit the maximum of peaks at $2.46 \mathrm{eV}(504 \mathrm{~nm})$ and $1.98 \mathrm{eV}(625 \mathrm{~nm})$, respectively, which correspond to blue-greenish and red emission, showing a blue-shift of $120 \mathrm{~nm}$ for the fluorinated polymer. A strong increase in the EL intensity of MEH-PPDFV compared to that of the non-fluorinated MEH-PPV is also evident, indicating a higher degree of twisting in the conjugated backbone caused by the $\mathrm{F}$ atoms, in agreement with theoretical predictions [34], which should reduce the interchain interactions in the solid state, thus increasing the EL efficiency. The EL of MEH-PPDFV is also quite stable, and after $30 \mathrm{~min}$ of continuous operation in air, only a slight decrease in intensity with no red-shift was observed [102].

It is worth noting that MEH-PPDFV shows a blue-shift in the absorption and comparable PL emission wavelength with respect to DA-PF, which is one of the most representative blue emitting conjugated polymers exhibiting a $\pi-\pi^{*}$ transition peak at $3.23 \mathrm{eV}(384 \mathrm{~nm})$ and a PL maximum ranging between $2.85(436 \mathrm{~nm})$ [104] and $2.7 \mathrm{eV}(460 \mathrm{~nm})$ [105]. On the contrary, the blue-greenish electroluminescent peak of MEH-PPDFV at $2.46 \mathrm{eV}$ is red shifted with respect to that of DA-PF at $2.9 \mathrm{eV}$, but still blue-shifted with respect to that of MA-PF at 2.25 eV [103] (Figure 11).

The optical properties of the perfluorinated polymers, 6F-PPV and 4F-PTV, in the solid state have also been investigated by spectroscopic ellipsometry [69]. Thin films of 6F-PPV and 4F-PTV were obtained by thermal evaporation under reduced pressure. The optical band gap, $\mathrm{E}_{\mathrm{g}}{ }^{\text {opt }}$, determined from the onset of absorption was found at $3.34 \mathrm{eV}$ for $\mathbf{6 F}-\mathbf{P P V}$ and $2.07 \mathrm{eV}$ for $\mathbf{4 F - P T V}$, while the maximum of the absorption, which corresponds to the HOMO-LUMO transition, was found at $4.18 \mathrm{eV}$ and $2.55 \mathrm{eV}$, respectively. By comparing the band gap values of the polymers, 6F-PPV and 4F-PTV, with those of the corresponding non-fluorinated polymers, the poly(1,4-phenylenevinylene) (PPV, band gap $=2.4 \mathrm{eV}$ [106]) and poly(2,5-thienylenevinylene) (PTV, band gap = $1.74 \mathrm{eV}$ [87]), a strong blue-shift of the optical band gap due to perfluorination can be observed, with the shift being more pronounced for 6F-PPV than for 4F-PTV. Interestingly, the polymer, 6F-PPV, shows a band gap value even higher than that of MEH-PPDFV. The reason of the strong blue-shift observed for 
6F-PPV and 4F-PTV may reside in several structural factors, such as reduced polymer chain length, the strong electron-withdrawing effect of fluorine atoms and steric repulsion between fluorine atoms on vinylene units and fluorine atoms ortho- to vinylene units on aromatic rings.

Figure 11. EL spectra of $80 \mathrm{~nm}$ thick films of MEH-PPDFV (blue curves) and MEH-PPV (red curves) recorded under identical experimental conditions using the device structure shown in the scheme in the inset. The MEH-PPDFV spectrum obtained after 30 min (dark green curve) of continuous operation is also shown. For comparison, the normalized EL spectra of an ITO/MA-PF/Al and of an ITO/DA-PF/A1 [103] (left vertical axis) are also shown, where MA-PF indicates a 9-monoalkylated polyfluorene and DA-PF indicates a 9,9-dialkyl polyfluorene. Reproduced with permission from [102]. Copyright 2009 Wiley-VCH Verlag GmbH \& Co. KGaA.

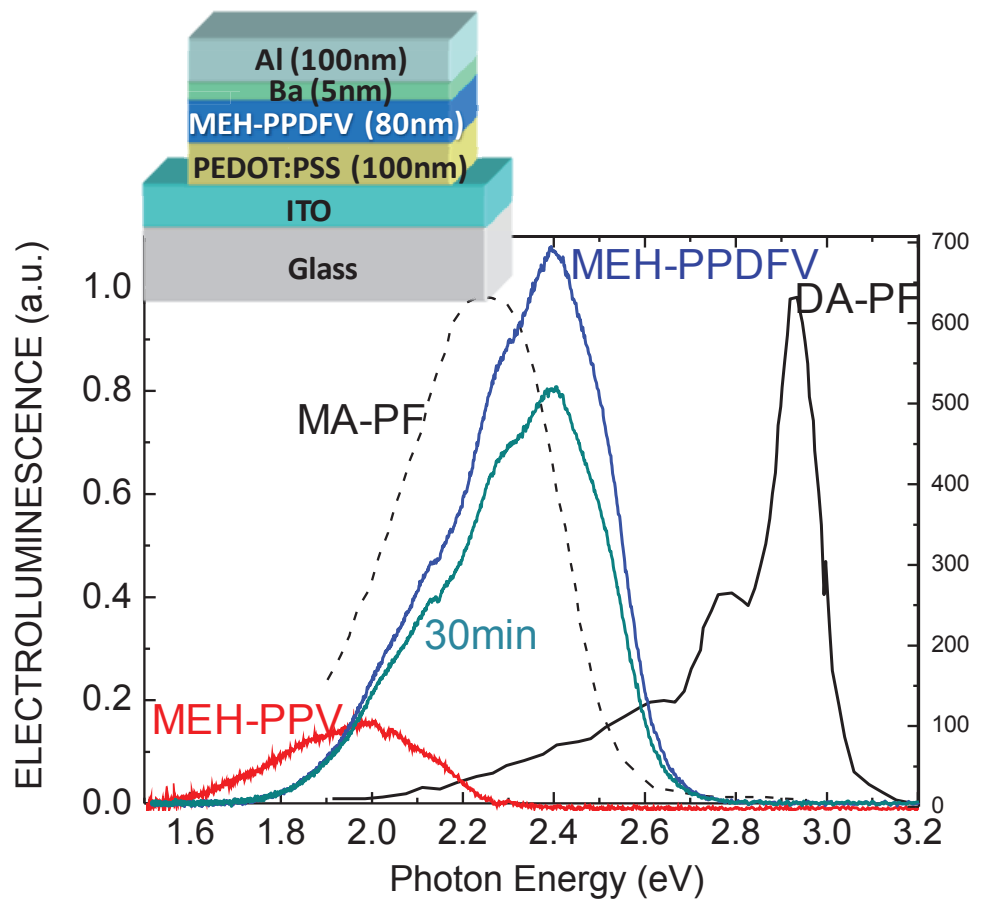

2.2.5. Photovoltaic Performance of PDTBTFV and PDTBTV in BHJ Solar Cells: Effects of Fluorination of Double Bonds

PDTBTFV and PDTBTV were used as donor materials in blend with PCBM in BHJ solar cells. Table 4 reports the photovoltaic performance recorded on non-optimized devices [99].

The devices fabricated with the fluorinated polymer PDTBTFV show, in general, better photovoltaic performance with significantly higher $\mathrm{V}_{\mathrm{OC}}$ and $\eta$ values compared to the non-fluorinated PDTBTV for all the measured weight ratios. Thermal annealing of the blend in the range $70-150{ }^{\circ} \mathrm{C}$ does not significantly affect the photovoltaic performances of both polymers, according to the data of the differential scanning calorimetry (DSC) analysis, which reveals the lack of any transition. Theoretical, electrochemical and optical characterization have been carried out in order to shed light on the interesting achievement of the capability of fluorine to enhance the photovoltaic properties of the polyconjugated structure investigated. The absorption spectra in chloroform solution of the two polymers PDTBTFV and PDTBTV are reported in Figure 12. 
Table 4. Photovoltaic performance of BHJ solar cells of PDTBTFV and PDTBTV in blend with PCBM: open circuit voltage, $\mathrm{V}_{\mathrm{OC}}$; short circuit current, $\mathrm{I}_{\mathrm{SC}}$; fill factor FF and power conversion efficiency, $\eta$.

\begin{tabular}{cccccc}
\hline Polymer & Polymer/PCBM Weight ratio & $\mathbf{V}_{\mathbf{O C}}[\mathbf{V}]$ & $\mathbf{I}_{\mathbf{S C}}\left[\mathbf{m A ~ \mathbf { ~ m } ^ { - 2 }}\right]$ & $\mathbf{F F}[\mathbf{\%}]$ & $\boldsymbol{\eta}[\mathbf{\%}]$ \\
\hline \multirow{3}{*}{ PDTBTFV } & $2: 7$ & 0.73 & 2.94 & 38.57 & 0.83 \\
& $3: 7$ & 0.75 & 3.55 & 37.97 & 1.05 \\
& $1: 1$ & 0.83 & 4.32 & 34.56 & 1.24 \\
\hline \multirow{3}{*}{ PDTBTV } & $2: 7$ & 0.55 & 3.33 & 28.83 & 0.53 \\
& $3: 7$ & 0.56 & 2.42 & 24.50 & 0.33 \\
& $1: 1$ & 0.43 & 3.15 & 33.51 & 0.46 \\
\hline
\end{tabular}

Figure 12. Normalized absorption spectra in chloroform solution of polymers PDTBTFV and PDTBTV.

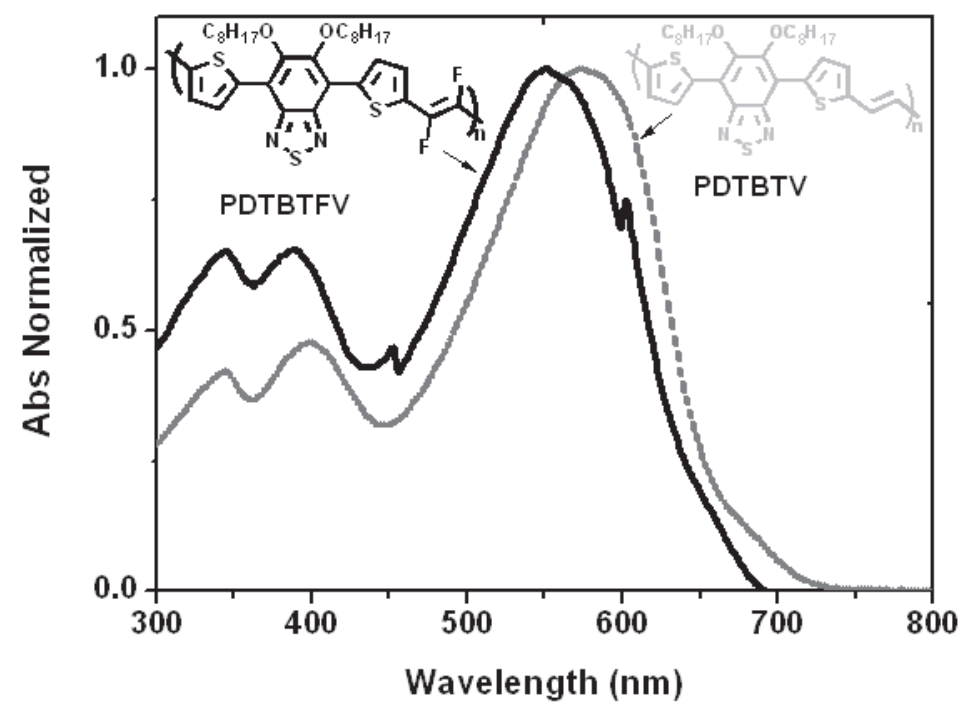

Both polymers show very wide absorption in the visible region, with maxima determined at $548 \mathrm{~nm}$ $(2.26 \mathrm{eV})$ for PDTBTFV and $569 \mathrm{~nm}(2.18 \mathrm{eV})$ for PDTBTV, resulting in a fluorination-induced blue-shift of $0.08 \mathrm{eV}$. Such blue-shift can be ascribed to the interplay of the mesomeric and inductive effect of fluorine atoms, with the latter effect dominating in the conjugated backbone of the investigate polymer structure, according to the results of theoretical TD-DFT calculations. The optical properties in the solid state have been investigated by spectroscopic ellipsometry, revealing the same trend in the absorption spectrum found in solution, with the optical band gap of the films determined at $1.79 \mathrm{eV}$ for PDTBTFV and $1.69 \mathrm{eV}$ for PDTBTV. In cyclic voltammetry, the oxidation and reduction processes have been investigated and the HOMO and LUMO energy levels estimated (based on Koopmans' theorem). The estimated HOMO energy levels are $-5.20 \mathrm{eV}$ for PDTBTFV and $-5.01 \mathrm{eV}$ for PDTBTV, while the LUMO energy levels are -3.14 for PDTBTFV and -3.03 for PDTBTV. Hence, the fluoro-functionalization of vinylene units results in an overall stabilization of both HOMO and LUMO energy levels, in agreement with data obtained from DFT calculations. The ellipsometric characterization discloses also a new remarkable effect of fluoro-functionalization of the double bonds, which is a significant enhancement of thin film light absorption in PDTBTFV compared to PDTBTV. This result can be attributed to a different packing of polymer chains in the solid state, with the 
fluorination of vinylene units being responsible for the increased intermolecular interactions between almost coplanar adjacent polymer chains, which, depending on the nature of the chains and interactions, can result in the observed increased absorption coefficient. When the polymers are blended with PCBM for the fabrication of BHJ solar cells, the absorption coefficient of the PDTBTFV-PCBM blend still remains higher than that of the PDTBTV-PCBM blend in the region of the main optical absorption of the polymer (Figure 13).

Figure 13. Ellipsometric spectra of the extinction coefficient and, in the inset, of the absorption coefficient for the fluorinated PDTBTFV and non-fluorinated PDTBTV polymers blended with phenyl-C61-butyric acid methyl ester (PCBM) (polymer/PCBM ratio 1:1). For comparison, the spectrum of PCBM is also shown.

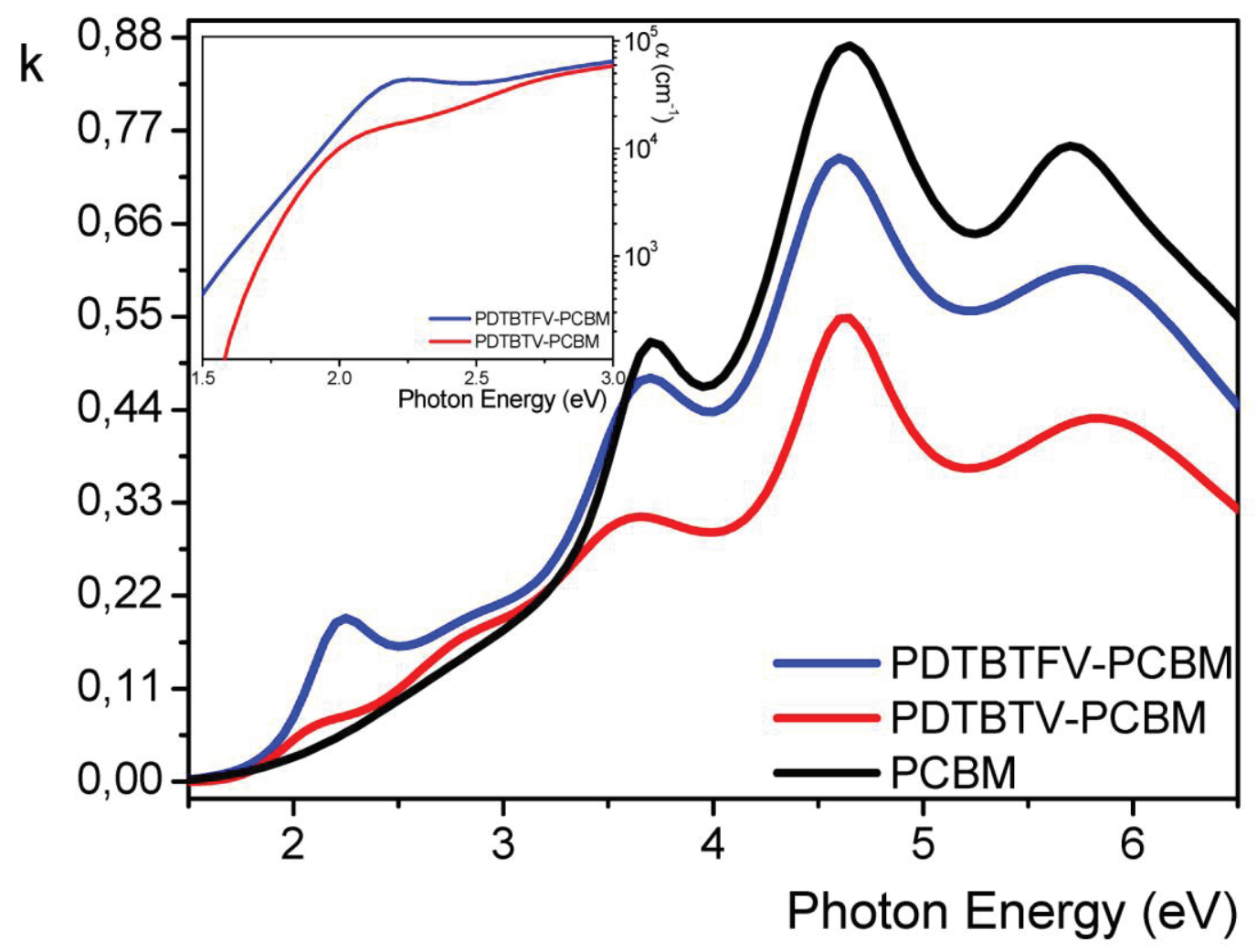

This enhancement of light absorption of the fluorinated polymer might be a main reason for the better photovoltaic performance of PDTBTFV compared to PDTBTV, since a higher number of free charge carriers is expected to be generated as a consequence of the higher light absorption. Summing up, the favorable effect of double bond fluorination on the photovoltaic performance of the poly(arylenevinylene) structure investigated can be attributed to a synergetic effect of (i) the lowering of HOMO and LUMO energy levels, leading to an increase in $\mathrm{V}_{\mathrm{OC}}$, and (ii) the increase in the molar extinction coefficient. The latter is very attractive from the perspective of reduction of BHJ film thickness, which should enhance the charge carrier generation at the interface and enables collection of carriers at the electrodes against their tendency to recombine. 


\section{Conclusions}

In summary, we have presented a series of arylenevinylene compounds, synthesized through the Stille cross-coupling reaction, selectively fluoro-functionalized on the aromatic ring and/or the vinylene units. Both oligomeric and polymeric materials have been prepared with a rigorous control of the conjugated molecular structure by an appropriate choice of monomers and experimental reaction conditions. The optical and electro-optical properties of the synthesized materials have been investigated in order to highlight the impact of fluorination on the properties, revealing electronic and steric effects that deeply affect the characteristics of the conjugated system. In many cases, fluorinated materials disclose enhanced properties with respect to the corresponding non-fluorinated materials, and better performance in electro-optical devices has been obtained. In particular, the fluorination of double bonds in PPVs with alkoxy substituents on the aromatic rings causes a strong blue shift of the optical properties, resulting in a new kind of blue-emitting polymers with enhanced EL and device stability. Simultaneously, the fluorination of double bonds in a PAV structure with low band gap significantly increases the light absorption capability in the solid state and, as a consequence, the photovoltaic performance in BHJ solar cells. The results referred to here show a special uncommon capability of fluorine to modulate optical and electrical properties of polyconjugated materials, offering a powerful tool in the hands of synthetic chemists for tailoring the properties of materials for specific applications.

\section{Acknowledgments}

This work was financially supported by the Italian National Council of Research (CNR), —Progto EFOR”. G. M. Farinola thanks Ministero dell'Istruzione, dell'Università e della Ricerca, USA (MIUR) and Università degli Studi di Bari Aldo Moro for financial support (Progetto PRIN 2009 PRAM8L).

\section{References and Notes}

1. Miller, R.D.; Chandross, E.A. 2010 Materials for Electronics. Chem. Rev. 2010, 110, 1-574.

2. Facchetti, A. p-Conjugated polymers for organic electronics and photovoltaic cell applications. Chem. Mater. 2011, 23, 733-758.

3. Nalwa, H.S. Handbook of Organic Electronics and Photonics; American Scientific Publishers: Stevenson Ranch, CA, USA, 2008.

4. Nguyen, T.P.; Destruel, P. Electroluminescent Devices Based on Organics and Conjugated Polymers. In Handbook of Luminescence, Display Materials and Devices; Nalwa, H.S., Rohwer, L.S., Eds.; American Scientific Publishers: Stevenson Ranch, CA, USA, 2003; Volume 1, pp. 1-129.

5. Kulkarni, A.P.; Tonzola, C.J.; Babel, A.; Jenekhe, S.A. Electron transport materials for organic light-emitting diodes. Chem. Mater. 2004, 16, 4556-4573.

6. Müllen, K.; Scherf, U. Organic Light-Emitting Devices: Synthesis, Properties and Applications; Wiley-VCH: Weinheim, Germany, 2006. 
7. Grimsdale, A.C.; Chan, K.L.; Martin, R.E.; Jokisz, P.G.; Holmes, A.B. Synthesis of light-emitting conjugated polymers for applications in electroluminescent devices. Chem. Rev. 2009, 109, 897-1091.

8. Singh, T.B.; Sariciftci, N.S.; Jaiswal, M.; Menon, R. Organic Field-Effect Transistors: From Materials to Device Physics. In Handbook of Organic Electronics and Photonics; Nalwa, H.S., Ed.; American Scientific Publishers: Stevenson Ranch, CA, USA, 2008.

9. Dimitrakopoulos, C.D.; Malenfant, P.R.L. Organic Thin Film Transistors for Large Area Electronics. Adv. Mater. 2002, 14, 99-117.

10. Thomas, S.W.; Joly, G.D.; Swager, T.M. Chemical sensors based on amplifying fluorescent conjugated polymers. Chem. Rev. 2007, 107, 1339-1386.

11. Brabec, C.; Dyakonov, V.; Parisi, J.; Sariciftci, N.S. Organic Photovoltaics: Concepts and Realization; Springer: Berlin, Germany, 2003.

12. Thompson, B.C.; Fréchet, J.M.J. Polymer-Fullerene composite solar cells. Angew. Chem. Int. Ed. 2008, 47, 58-77.

13. Günes, S.; Neugebauer, H.; Sariciftci, N.S. Conjugated polymer-based organic solar cells. Chem. Rev. 2007, 107, 1324-1338.

14. Babudri, F.; Farinola, G.M.; Naso, F.; Ragni, R. Fluorinated organic materials for electronic and optoelectronic applications: The role of fluorine atom. Chem. Commun. 2007, 1003-1022.

15. Farinola, G.M.; Cardone, A.; Babudri, F.; Martinelli, C.; Naso, F.; Bruno, G.; Losurdo, M. Fluorinated Poly( $p$-phenylenevinylene)s: Synthesis and optical properties of an intriguing class of luminescent polymers. Materials 2010, 3, 3077-3091.

16. Cardone, A.; Martinelli, C.; Babudri, F.; Naso, F.; Pinto, V.; Farinola, G.M. Synthesis of fluorinated (electro)luminescent arylenevinylene polymers and oligomers. Curr. Org. Synth. 2012, 9, 150-162.

17. Facchetti, A.; Deng, Y.; Wang, A.; Koide, Y.; Sirringhaus, H.; Marks, T.J.; Friend, R.H. Tuning the semiconducting properties of sexithiophene by $\alpha, \omega$-substitution- $\alpha, \omega$-diperfluorohexylsexithiophene: the first n-type sexithiophene for thin film transistors. Angew. Chem. Int. Ed. 2000, 39, 4547-4551.

18. Facchetti, A.; Yoon, M.-H.; Stern, C.L.; Hutchinson, G.R.; Ratner, M.A.; Marks, T.J. Building blocks for n-type molecular and polymeric electronics. Perfluoroalkyl- versus alkyl-functionalized oligothiophenes ( $\mathrm{nTs} ; \mathrm{n}=2-6)$. Systematic synthesis, spectroscopy, electrochemistry, and solid-state organization. J. Am. Chem. Soc. 2004, 126, 13480-13501.

19. Sakamoto, Y.; Komatsu, S.; Suzuki, T. Tetradecafluorosexithiophene: The first perfluorinated oligothiophene. J. Am. Chem. Soc. 2001, 123, 4643-4644.

20. Newman, C.R.; Frisbie, C.D.; da Silva Filho, D.A.; Brédas, J.-L.; Ewbank, P.C.; Mann, K.R. Introduction to organic thin film transistors and design of n-channel organic semiconductors. Chem. Mater. 2004, 16, 4436-4451.

21. Usta, H.; Facchetti, A.; Marks, T.J. n-Channel Semiconductor Materials Design for Organic Complementary Circuits. Acc. Chem. Res. 2011, 44, 501-510.

22. Wen, Y.; Liu, Y. Recent progress in n-channel organic thin-film transistors. Adv. Mater. 2010, $22,1331-1345$. 
23. Kryachko, E.; Scheiner, S. C-H・ F Hydrogen bonds. Dimers of fluoromethanes. J. Phys. Chem. A 2004, 108, 2527-2535.

24. Müllen, K.; Wegner, G. Electronic Materials: The Oligomer Approach; Wiley-VCH: Weinheim, Germany, 1998.

25. He, F.; Tian, L.; Xie, W.; Li, M.; Gao, Q.; Hanif, M.; Zhang, Y.; Cheng, G.; Yang, B.; Ma, Y.; et al. Highly efficient blue organic light emitting devices based on improved guest/host combination. J. Phys. Chem. C 2008, 112, 12024-12029.

26. Sander, R.; Stümpflen, V.; Wendorff, J.H.; Greiner, A. Synthesis, properties, and guest-host systems of triphenylamine-based oligo(arylene-vinylene)s: Advanced materials for led applications. Macromolecules 1996, 29, 7705-7708.

27. Peeters, E.; van Hal, P.A.; Knol, J.; Bramec, C.J.; Sariciftci, N.S.; Hummelen, J.C.; Janssen, R.A.J. Synthesis, photophysical properties, and photovoltaic devices of oligo( $p$-phenylene vinylene)-fullerene dyads. J. Phys. Chem. B 2000, 104, 10174-10190.

28. Neuteboom, E.E.; Meskers, S.C.J.; van Hal, P.A.; van Duren, J.K.J.; Meijer, E.W.; Janssen, R.A.J.; Dupin, H.; Pourtois, G.; Cornil, J.; Lazzaroni, R.; et al. Alternating oligo(p-phenylene vinylene)-perylene bisimide copolymers: Synthesis, photophysics, and photovoltaic properties of a new class of donor-acceptor materials. J. Am. Chem. Soc. 2003, 125, 8625-8638.

29. Jørgensen, M.; Krebs, F.C. Stepwise unidirectional synthesis of oligo phenylene vinylenes with a series of monomers: Use in plastic solar cells. J. Org. Chem. 2005, 70, 6004-6017.

30. Babudri, F.; Cardone, A.; de Cola, L.; Farinola, G.M.; Kottas, G.S.; Martinelli, C.; Naso, F. Synthesis of oligoarylenevinylenes with fluorinated double bonds. Synthesis 2008, 1580-1588.

31. Xue, L.; Lu, L.; Pedersen, S.D.; Liu, Q.; Narske, R.M.; Burton, D.J. A novel stereospecific route to (E)- and (Z)-(2-substituted-1,2-difluoroethenyl) stannanes. J. Org. Chem. 1997, 62, 1064-1071.

32. Burton, D.J.; Liu, Q. Stereoselective preparation of (E)-(1,2-difluoro-1,2-ethenediyl) bis[tributylstannane] and stereospecific synthesis of (E)-1,2-difluorostilbenes. Org. Lett. 2002, 4, $1483-1485$.

33. Eaton, D.F. Reference materials for fluorescence measurements. Pure Appl. Chem. 1988, 60, $1107-1114$.

34. Piacenza, M.; Della Sala, F.; Farinola, G.M.; Martinelli, C.; Gigli, G. Large blue-shift in the optical spectra of fluorinated polyphenylenevinylenes. A combined theoretical and experimental study. J. Phys. Chem. B 2008, 112, 2996-3004.

35. Bettini, S.; Valli, L.; Santino, A.; Martinelli, C.; Farinola, G.M.; Cardone, A.; Sgobba, V.; Giancane, G. Spectroscopic investigations, characterization and chemical sensor application of composite Langmuir-Schäfer films of anthocyanins and oligophenylenevinylene derivatives. Dyes Pigment. 2012, 94, 156-162.

36. Torskangerpoll, K.; Andersen, O.M. Colour stability of anthocyanins in aqueous solutions at various pH values. Food Chem. 2005, 89, 427-440.

37. Burroughes, J.H.; Bradley, D.D.C.; Brown, A.R.; Marks, R.N.; Mackay, K.; Friend, R.H.; Burn, P.L.; Holmes, A.B. Light-emitting diodes based on conjugated polymers. Nature 1990, 347, 539-541. 
38. Gilch, H.G.; Wheelwright, W.L. Polymerization of $\alpha$-halogenated $p$-xylenes with base. J. Polym. Sci. A Polym. Chem. 1966, 4, 1337-1349.

39. Wessling, R.A. The polymerization of xylylene bisdialkyl sulfonium salts. J. Polym. Sci. Polym. Symp. 1985, 72, 55-66.

40. Gourley, K.D.; Lillya, C.P.; Reynolds, J.R.; Chien, J.C.W. Electrically conducting polymers: Arsenic pentafluoride-doped poly(phenylenevinylene) and its analogs. Macromolecules 1984, 17, $1025-1033$.

41. Chen, Z.-K.; Meng, H.; Lai, Y.-H.; Huang, W. Photoluminescent Poly(p-phenylenevinylene)s with an aromatic oxadiazole moiety as the side chain: Synthesis, electrochemistry and spectroscopy study. Macromolecules 1999, 32, 4351-4358.

42. Ahn, T.; Song, S.-Y.; Shim, H.-K. Highly photoluminescent and blue-green electroluminescent polymers: New silyl- and alkoxy-substituted Poly( $p$-phenylenevinylene) related copolymers containing carbazole or fluorene groups. Macromolecules 2000, 33, 6764-6771.

43. Greenham, N.C.; Moratti, S.C.; Bradley, D.D.C.; Friend, R.H.; Holmes, A.B. Efficient light-emitting diodes based on polymers with high electron affinities. Nature 1993, 365, $628-630$.

44. Moratti, S.C.; Cervini, R.; Holmes, A.B.; Baigent, D.R.; Friend, R.H.; Greenham, N.C.; Grüner, J.; Hamer, P.J. High electron affinity polymers for LEDs. Synth. Met. 1995, 71, 2117-2120;

45. Chen, S.-A.; Chang, E.-C. Structure and Properties of Cyano-Substituted Poly(2,5-dialkoxy-p-phenylene vinylene)s. Macromolecules 1998, 31, 4899-4907;

46. Jin, Y.; Ju, J.; Kim, J.; Lee, S.; Kim, J.Y.; Park, S.H.; Son, S.-M.; Jin, S.-H.; Lee, K.; Suh, H. Design, synthesis and electroluminescent property of CN-Poly(dihexylfluorenevinylene) for LEDs. Macromolecules 2003, 36, 6970-6975.

47. Babudri, F.; Farinola, G.M.; Naso, F. Organometallic chemistry directed towards the synthesis of electroactive materials: Stereoselective routes to extended polyconjugated systems. Pure Appl. Chem. 1999, 71, 1485-1492.

48. Babudri, F.; Farinola, G.M.; Naso, F. Synthesis of conjugated oligomers and polymers: The organometallic way. J. Mater. Chem. 2004, 14, 11-34.

49. Farinola, G.M.; Babudri, F.; Cardone, A.; Hassan Omar, O.; Naso, F. Sinthesis of substituted conjugated polymers: Tuning properties by functionalization. Pure Appl. Chem. 2008, 80, $1735-1746$.

50. Babudri, F.; Cicco, S.R.; Chiavarone, L.; Farinola, G.M.; Lopez, L.C.; Naso, F.; Scamarcio, G. Synthesis and optical investigations of low molecular weight alkoxy-substituted poly(p-phenylenevinylene)s. J. Mater. Chem. 2000, 10, 1573-1579.

51. Brooke, G.M.; Mawson, S.D. The attempted synthesis of polyparatetrafluorophenylene vinylene via water-soluble and organic solvent-soluble precursor polymers. J. Fluor. Chem. 1990, 50, 101-109.

52. Brooke, G.M.; Mawson, S.D. Oligomeric polyparatetrafluorophenylene vinylenes: A new synthesis based on the nucleophilic substitution of the para fluorine during the reaction of (E)-2-(pentafluorophenyl)ethenyl lithium with hexafluorobenzene. J. Fluor. Chem. 1990, 50, 101-109. 
53. Babudri, F.; Cardone, A.; Chiavarone, L.; Ciccarella, G.; Farinola, G.M.; Naso, F.; Scamarcio, G. Synthesis and characterization of poly(2,3,5,6-tetrafluoro-1,4-phenylenevinylene). Chem. Commun. 2001, 1940-1941.

54. Farina, V. New perspective in the cross-coupling reactions of organostannanes. Pure Appl. Chem. 1996, 68, 73-78.

55. Skelton, R.; Dubois, F.; Zenobi, R.A. MALDI sample preparation method suitable for insoluble polymers. Anal. Chem. 2000, 72, 1707-1710.

56. Pinto, M.R.; Hu, B.; Karasz, F.E.; Akcelrud, L. Emitting polymers containing cyano groups. Synthesis and photophysical properties of a fully conjugated polymer obtained by Wittig reaction. Polymer 2000, 41, 8095-8102.

57. Xiao, Y.; Yu, W.-L.; Pei, J.; Chen, Z.; Huang, W.; Heeger, A.J. A Novel Series of Copolymers Containing 2,5-Dicyano-1,4-phenylenevinylene-synthetic tuning of the HOMO and LUMO energy levels of conjugated polymers. Chem. Eur. J. 2000, 6, 1318-1321.

58. Riehn, R.; Morgado, J.; Iqbal, R.; Moratti, S.C.; Holmes, A.B.; Volta, S.; Cacialli, F. Electrochemical and electroluminescent properties of random copolymers of fluorine- and alkoxysubstituted poly(p-phenylene vinylene)s. Macromolecules 2000, 33, 3337-3341.

59. Farinola, G.M.; Cassano, T.; Tommasi, R.; Babudri, F.; Cardone, A.; Naso, F. Third-order nonlinear optical properties of copoly(2,3,5,6-tetrafluoro-1,4-phenylenevinylene-2,5-dialkoxy1,4-phenylenevinylene)s, a novel class of push-pull substituted PPVs. Proc. SPIE Int. Soc. Opt. Eng. 2001, 4461, 296-303.

60. Cassano, T.; Tommasi, R.; Babudri, F.; Cardone, A.; Farinola, G.M.; Naso, F. High third-order nonlinear optical susceptibility in new fluorinated poly( $p$-phenylenevinylene) copolymers measured with the Z-scan technique. Opt. Lett. 2002, 27, 2176-2178.

61. Babudri, F.; Cardone, A.; Farinola, G.M.; Naso, F.; Cassano, T.; Chiavarone, L.; Tommasi, R. Synthesis and optical properties of a copolymer of tetrafluoro- and dialkoxy-substituted poly( $p$-phenylenevinylene) with a high percentage of fluorinated units. Macromol. Chem. Phys. 2003, 204, 1621-1627.

62. Benjamin, I.; Faraggi, E.Z.; Avny, Y.; Davidov, D.; Neumann, R. Fluorinated poly(pphenylenevinylene) copolymers: Preparation and use in light-emitting diodes. Chem. Mater. 1996, 8, 352-355.

63. Tommasi, R.; Cassano, T.; Farinola, G.M.; Babudri, F.; Cardone, A.; Naso, F. Nonlinear Optical Measurements in Copoly(2,3,5,6-tetrafluoro-1,4-phenylenevinylene-2,5-dialkoxy-1,4-phenylenevinylene)s using the Z-scan Technique. In Nonlinear Optics: Materials, Fundamentals and Applications; Sawchuk, A., Ed.; Optical Society of America: Wangshington, DC, USA, 2002; pp. 269-271.

64. Yohannes, T.; Neugebauer, H.; Farinola, G.M.; Winder, C.; Babudri, F.; Cardone, A.; Naso, F.; Sariciftci, N.S. Vibrational spectroscopic study of a push-pull substituted fluorinated poly (p-phenylenevinylene) copolymer. Synth. Met. 2005, 152, 149-152.

65. Jin, Y.; Kim, J.; Lee, S.; Kim, J.Y.; Park, S.H.; Lee, K.; Suh, H. Novel electroluminescent polymers with fluoro groups in vinylene units. Macromolecules 2004, 37, 6711-6715.

66. Jin, Y; Jee J; Kim, K; Kim, J; Song, S; Park, S.H.; Lee, K.; Suh, H. Synthesis and electroluminescent properties of copolymers based on PPV with fluoro groups in vinylene units. Polymer 2007, 48, 1541-1549. 
67. Becker, H.; Spreitzer, H.; Ibrom, K.; Kreuder, W. New insights into the microstructure of Gilch-polymerized PPVs. Macromolecules 1999, 32, 4925-4932.

68. Babudri, F.; Cardone, A.; Farinola, G.M.; Martinelli, C.; Mendichi, R.; Naso, F.; Striccoli, M. Synthesis of poly(arylenevinylene)s with fluorinated vinylene units. Eur. J. Org. Chem. 2008, 1977-1982.

69. Cardone, A.; Martinelli, C.; Pinto, V.; Babudri, F.; Losurdo, M.; Bruno, G.; Pinalysa, C.; Naso, F.; Farinola, G.M. Synthesis and characterization of perfluorinated arylenevinylene polymers. J. Poly. Sci. A Polym. Chem. 2010, 48, 285-291.

70. Liang, Y.; Yu, L. A New class of semiconducting polymers for bulk heterojunction solar cells with exceptionally high performance. Acc. Chem. Res. 2010, 43, 1227-1236.

71. Liang, Y.; Feng, D.; Wu, Y.; Tsai, S.-T.; Li, G.; Ray, C.; Yu, L. Highly efficient solar cell polymers developed via fine-tuning of structural and electronic properties. J. Am. Chem. Soc. 2009, 131, 7792-7799.

72. Schroeder, B.C.; Huang, Z.G.; Ashraf, R.S.; Smith, J.; D'Angelo, P.; Watkins, S.E.; Anthopoulos, T.D.; Durrant, J.R.; McCulloch, I. Silaindacenodithiophene-based low band gap polymers-The effect of fluorine substitution on device performances and film morphologies. Adv. Funct. Mater. 2012, 22, 1663-1670.

73. Price, S.C.; Steuart, A.C.; Yang, L.; Zhou, H.; You, W. Fluorine substituted conjugated polymer of medium band gap yields $7 \%$ efficiency in polymer-fullerene solar cells. J. Am. Chem. Soc. 2011, 133, 4625-4631.

74. Yu, G.; Gao, J.; Hummelen, J.C.; Wudl, F.; Heeger, A.J. Polymer photovoltaic cells: Enhanced efficiencies via a network of internal donor-acceptor heterojunctions. Science 1995, 270, 1789-1791.

75. Halls, J.J.M.; Walsh, C.A.; Greenham, N.C.; Marseglia, E.A.; Friend, R.H.; Moratti, S.C.; Holmes, A.B. Efficient photodiodes from interpenetrating polymer networks. Nature 2002, 376, 498-500.

76. Thompson, B.C.; Kim, Y.G.; Reynolds, J.R. Spectral broadening in MEH-PPV:PCBM-based photovoltaic devices via blending with a narrow band gap cyanovinylene-dioxythiophene polymer. Macromolecules 2005, 38, 5359-5362.

77. Tajima, K.; Suzuki, Y.; Hashimoto, K. Polymer photovoltaic devices using fully regioregular poly[(2-methoxy-5-(3',7'-dimethyloctyloxy))-1,4-phenylenevinylene]. J. Phys. Chem. C 2008, $112,8507-8510$.

78. Shaheen, S.E.; Brabec, C.J.; Sariciftci, N.S.; Padinger, F.; Fromherz, T.; Hunnelen, J.C. 2.5\% efficient organic plastic solar cells. Appl. Phys. Lett. 2001, 78, 841-843.

79. Egbe, D.A.M.; Bader, C.; Klemm, E.; Ding, L.; Karasz, F.E.; Grummt, U.-W.; Birckner, E. Influence of the conjugation pattern on the photophysical properties of Alkoxy-substituted PE/PV hybrid polymers. Macromolecules 2003, 36, 9303-9312.

80. van Duren, J.K.J.; Yang, X.; Loos, J.; Bulle-Lieuwma, C.W.T.; Sieval, A.B.; Hummelen, J.C.; Janssen, R.A.J. Relating the morphology of $\operatorname{poly}(p$-phenylene vinylene)/methanofullerene blends to solar-cell performance. Adv. Funct. Mater. 2004, 14, 425-434. 
81. Sariciftci, N.S.; Braun, D.; Zhang, C.; Sardanov, V.; Heeger, A.J.; Stucky, G.; Wudl, F. Semiconducting polymer-buckminsterfullerene heterojunctions: Diodes, photodiodes, and photovoltaic cells. Appl. Phys. Lett. 1993, 62, 585-587.

82. Halls, J.J.M.; Friend, R.H. The photovoltaic effect in a poly( $p$-phenylenevinylene $) /$ perylene heterojunction. Synth. Met. 1997, 85, 1307-1308.

83. Sariciftci, N.S.; Smilowitz, L.; Heeger, A.J.; Wudl, F. Photoinduced electron transfer from a conducting polymer to buckminsterfullerene. Science 1992, 258, 1474-1476.

84. Henckens, A.; Knipper, M.; Polec, I.; Manca, J.; Lutsen, L.; Vanderzande, D. Poly(thienylene vinylene) derivatives as low band gap polymers for photovoltaic applications. Thin Solid Films 2004, 451-452, 572-579.

85. Smith, A.P.; Smith, R.R.; Taylor, B.E.; Durstock, M.F. An investigation of Poly(thienylene vinylene) in organic photovoltaic devices. Chem. Mater. 2004, 16, 4687-4692;

86. Huo, L.; Chen, T.L.; Zhou, Y.; Hou, J.; Chen, H.-Y.; Yang, Y.; Li, Y. Improvement of photoluminescent and photovoltaic properties of poly(thienylene vinylene) by carboxylate substitution. Macromolecules 2009, 42, 4377-4380.

87. Fuchigami, H.; Tsumura, A.; Koezuka, H. Polythienylenevinylene thin-film transistor with high carrier mobility. Appl. Phys. Lett. 1993, 63, 1372-1374.

88. Wan, M.; Wu, W.; Sang, G.; Zou, Y.; Liu, Y.; Li, Y. Poly(thienylene-vinylene-thienylene) with cyano substituent: Synthesis and application in field-effect transistor and polymer solar cell. J. Polym. Sci. A Polym. Chem. 2009, 47, 4028-4036.

89. Thompson, B.C.; Kim, Y.-G.; McCarley, T.D.; Reynolds, J.R. Soluble narrow band gap and blue propylenedioxythiophene-cyanovinylene polymers as multifunctional materials for photovoltaic and electrochromic applications. J. Am. Chem. Soc. 2006, 128, 12714-12725.

90. Colladet, K.; Fourier, S.; Cleij, T.J.; Lusten, L.; Gelan, J.; Vanderzande, D.; Nguyen, L.H.; Neugebauer, H.; Sariciftci, S.; Aguirre, A.; et al. Low band gap donor-acceptor conjugated polymers toward organic solar cells applications. Macromolecules 2007, 40, 65-72.

91. Galand, E.M.; Kim, Y.-G.; Mwaura, J.K.; Jones, A.G.; McCarley, T.D.; Shrotriya, V.; Yang, Y.; Reynolds, J.R. Optimization of narrow band-gap propylenedioxythiophene: cyanovinylene copolymers for optoelectronic applications. Macromolecules 2006, 39, 9132-9142.

92. Mei, J.; Heston, N.C.; Vasilyeva, S.V.; Reynolds, J.R.A Facile approach to defect-free vinylenelinked benzothiadiazole-thiophene low-bandgap conjugated polymers for organic electronics. Macromolecules 2009, 42, 1482-1487.

93. Beaujuge, P.M.; Vasilyeva, S.V.; Ellinger, S.; McCarley, T.D.; Reynolds, J.R. Unsaturated linkages in dioxythiophene-benzothiadiazole donor-acceptor electrochromic polymers: The key role of conformational freedom. Macromolecules 2009, 42, 3694-3706.

94. Liu, B.; Najari, A.; Pan, C.; Leclerc, M.; Xiao, D.; Zou, Y. New low bandgap dithienylbenzothiadiazole vinylene based copolymers: synthesis and photovoltaic properties. Macromol. Rapid Commun. 2010, 31, 391-398.

95. Li, Y.; Xue, L.; Li, H.; Li, Z.; Xu, B.; Wen, S.; Tian, W. Energy Level and Molecular Structure Engineering of conjugated donor-acceptor copolymers for photovoltaic applications. Macromolecules 2009, 42, 4491-4499. 
96. Wen, S.; Pei, J.; Zhou, Y.; Li, P.; Xue, L.; Li, Y.; Xu, B.; Tian, W. Synthesis of 4,7-Diphenyl-2,1,3-Benzothiadiazole-based copolymers and their photovoltaic applications. Macromolecules 2009, 42, 4977-4984.

97. Abbotto, A.; Calderon, E.H.; Dangate, M.S.; de Angelis, F.; Manfredi, N.; Mari, C.M.; Marinzi, C.; Mosconi, E.; Muccini, M.; Ruffo R.; et al. Pyridine-EDOT heteroarylene-vinylene donor-acceptor polymers. Macromolecules 2010, 43, 9698-9713.

98. Abbotto, A.; Seri, M.; Dangate, M.S.; de Angelis, F.; Manfredi, N.; Mosconi, E.; Bolognesi, M.; Ruffo, R.; Salamone, M.M.; Muccini, M. A vinylene-linked benzo[1,2-b:4,5-b]dithiophene2,1,3-benzothiadiazole low-bandgap polymer. J. Polym. Sci. A Polym. Chem. 2012, 50, 2829-2840.

99. Cardone, A.; Martinelli, C.; Losurdo, M.; Dilonardo, E.; Bruno, G.; Scavia, G.; Destri, S.; Cosma, P.; Salamandra, L.; Reale, A.; et al. Fluoro-Functionalization of vinylene units in a polyarylenevinylene for polymer solar cells. J. Mater. Chem. A 2013, 1, 715-727.

100. Piacenza, M.; Comoretto, D.; Burger, M.; Morandi, V.; Marabelli, F.; Martinelli, C.; Farinola, G.M.; Cardone, A.; Gigli, G.; Della Sala, F. Raman spectra of poly( $p$-phenylenevinylene)s with fluorinated vinylene units: Evidence of inter-ring distortion. ChemPhysChem 2009, 10, 1284-1290.

101. Losurdo, M.; Giangregorio, M.M.; Capezzuto, P.; Bruno, G.; Babudri, F.; Cardone, A.; Martinelli, C.; Farinola, G.M.; Naso, F.; Büchel, M. Impact of fluorinated vinylene units on supramolecular organization and optical properties of poly( $p$-phenylenedifluorovinylene) thin films as a class of blue band gap conjugated polymers. Polymer 2008, 49, 4133-4140.

102. Losurdo, M.; Giangregorio, M.M.; Capezzuto, P.; Cardone, A.; Martinelli, C.; Farinola, G.M.; Babudri, F.; Naso, F.; Büchel, M.; Bruno, G. Blue-gap poly(p-phenylenevinylene)s with fluorinated double bonds: interplay between supramolecular organization and optical properties in thin films. Adv. Mater. 2009, 21, 1115-1120.

103. Scherf, U.; List, E.J.W. Semiconducting polyfluorenes-Towards reliable structure-property relationships. Adv. Mater. 2002, 14, 477-487.

104. Grice, A.W.; Bradley, D.D.C.; Bernius, M.T.; Inbasekaran, M.; Wu, W.W.; Woo, E.P. High brightness and efficiency blue light-emitting polymer diodes. Appl. Phys. Lett. 1998, 73, 629-631.

105. Parker, L.D.; Pei, Q.; Marrocco, M. Efficient blue electroluminescence from a fluorinated polyquinoline. Appl. Phys. Lett. 1994, 65, 1272-1274.

106. Leuze, M.; Hohloch, M.; Hanack, M. Electrochemical investigation on PPV model compounds. Chem. Mater. 2002, 14, 3339-3342.

(C) 2013 by the authors; licensee MDPI, Basel, Switzerland. This article is an open access article distributed under the terms and conditions of the Creative Commons Attribution license (http://creativecommons.org/licenses/by/3.0/). 